\title{
The noncoding MIR100HG RNA enhances the autocrine function of transforming growth factor $\beta$ signaling
}

\author{
Panagiotis Papoutsoglou $\mathbb{1}^{1,2}$ - Dorival Mendes Rodrigues-Junior ${ }^{1} \cdot$ Anita Morén $^{1} \cdot$ Andrew Bergman $^{1}$. \\ Fredrik Pontén $\mathbb{D}^{3} \cdot$ Cédric Coulouarn $\mathbb{D}^{2} \cdot$ Laia Caja $\mathbb{D}^{1} \cdot$ Carl-Henrik Heldin $\mathbb{D}^{1} \cdot$ Aristidis Moustakas $\mathbb{( I D}^{1}$
}

Received: 8 July 2019 / Revised: 31 March 2021 / Accepted: 15 April 2021 / Published online: 4 May 2021

(c) The Author(s) 2021. This article is published with open access

\begin{abstract}
Activation of the transforming growth factor $\beta$ (TGF $\beta$ ) pathway modulates the expression of genes involved in cell growth arrest, motility, and embryogenesis. An expression screen for long noncoding RNAs indicated that TGF $\beta$ induced mir-100let-7a-2-mir-125b-1 cluster host gene $(M I R 100 H G)$ expression in diverse cancer types, thus confirming an earlier demonstration of TGF $\beta$-mediated transcriptional induction of MIRIOOHG in pancreatic adenocarcinoma. MIRIOOHG depletion attenuated TGF $\beta$ signaling, expression of TGF $\beta$-target genes, and TGF $\beta$-mediated cell cycle arrest. Moreover, MIRIOOHG silencing inhibited both normal and cancer cell motility and enhanced the cytotoxicity of cytostatic drugs. MIR100HG overexpression had an inverse impact on TGF $\beta$ signaling responses. Screening for downstream effectors of MIRIOOHG identified the ligand TGF 31 . MIRIOOHG and TGFB1 mRNA formed ribonucleoprotein complexes with the RNA-binding protein HuR, promoting TGF $\beta 1$ cytokine secretion. In addition, TGF $\beta$ regulated let-7a-2-3p, miR-125b-5p, and $m i R-125 b-1-3 p$ expression, all encoded by MIR100HG intron-3. Certain intron-3 miRNAs may be involved in TGF $\beta /$ SMAD-mediated responses (let-7a-2-3p) and others $(m i R-100, m i R-125 b)$ in resistance to cytotoxic drugs mediated by MIRIOOHG. In support of a model whereby TGF $\beta$ induces MIR100HG, which then enhances TGF $\beta 1$ secretion, analysis of human carcinomas showed that MIRIOOHG expression correlated with expression of TGFBI and its downstream extracellular target TGFBI. Thus, MIRIOOHG controls the magnitude of TGF $\beta$ signaling via TGF $\beta 1$ autoinduction and secretion in carcinomas.
\end{abstract}

\section{Introduction}

Parts of the human genome are transcribed into noncoding RNAs (ncRNAs) that regulate gene expression, and have little or no protein-coding potential, such as microRNAs

Supplementary information The online version contains supplementary material available at https://doi.org/10.1038/s41388021-01803-8.

Aristidis Moustakas

aris.moustakas@imbim.uu.se

1 Department of Medical Biochemistry and Microbiology, Science for Life Laboratory, Box 582, Biomedical Center, Uppsala University, Uppsala, Sweden

2 InInserm, Univ Rennes, UMR_S 1242, COSS (Chemistry, Oncogenesis Stress Signaling), Centre de Lutte contre le Cancer Eugène Marquis, Rennes, France

3 Department of Immunology, Genetics and Pathology, Box 256, Rudbeck Laboratory, Uppsala University, Uppsala, Sweden
(miRNAs) [1] and long ncRNAs (lncRNAs) [2, 3]. Upon cleavage during splicing, either intronic or exonic regions of lncRNAs, called miRNA host genes, can give rise to miRNAs [2, 3], such as miR-17-92a-1 cluster host gene (MIRI7HG) [4], miR-31 host gene (MIR3IHG) [5], and $m i R$ 100-let-7a-2-miR-125b-1 cluster host gene (MIR100HG) [6].

MiRNAs inhibit translation or induce degradation of mRNAs during development [7], and control proliferation and migration, which explains their deregulated expression in cancer [8]. MiR17-92 favors cell cycle entry by targeting the cell cycle inhibitor $\mathrm{p} 21^{\mathrm{Cip} 1}$ and retinoblastoma family proteins [8]. The let-7 family targets cyclins and cyclindependent kinases (CDKs), while diverse cancers downregulate let-7 expression [8]. MiR-125b targets CDK6 and other cell cycle regulators, acting as an antiproliferative miRNA [8]. MiRNAs may exert context-dependent actions in cancer, when viewed as units of signaling pathways.

One such pathway is transforming growth factor $\beta$ (TGF $\beta$ ) that signals via membrane receptors, which activate effector transcription factors (SMADs) and mitogen- 
activated protein kinases (MAPKs), to regulate target genes that control the cell cycle, migration, extracellular matrix remodeling, and epithelial-mesenchymal transition (EMT) [9]. Such target genes of TGF $\beta$ can be proteincoding or noncoding [10]. In normal or benign hyperplastic epithelial cells, TGF $\beta$ arrests the cell cycle and suppresses tumorigenesis by transcriptionally inducing the CDK inhibitors $\mathrm{p} 15^{\mathrm{Ink} 4 \mathrm{~b}}, \mathrm{p} 21^{\mathrm{Cip} 1}$, and $\mathrm{p} 27^{\mathrm{Kip} 1}$, and repressing the proto-oncogene $c-M Y C$ [11]. In advanced tumors, TGF $\beta$ promotes stemness, invasiveness, and metastasis [9]. For example, TGF $\beta$ induces MIR100HG expression in pancreatic tumors, generating $m i R-100$ and $m i R-125 b-1$, which act in a protumorigenic manner, and let-7a-2, which acts oppositely [6].

We screened for TGF $\beta$-regulated IncRNAs in human keratinocytes and observed upregulation of MIRIOOHG [12], which was validated in diverse cell types. We then established a role of MIRIOOHG in TGF $\beta$ autoinduction, a central feature in TGF $\beta$ biology, especially in the context of cancer.

\section{Results}

\section{TGF $\beta$ receptor-SMAD signaling induces MIR100HG expression in diverse normal and cancer cells}

We searched for lncRNAs whose expression is regulated by the TGF $\beta$ pathway [12]. Microarray analysis in human $\mathrm{HaCaT}$ keratinocytes stimulated with TGF $\beta 1$ for early, intermediate, or long time periods, detected several up- or downregulated lncRNA genes (Fig. 1a, b). The screen was completed by selecting 23 TGF $\beta$-regulated IncRNAs, silencing their expression by short-interfering (si) RNAs, and by monitoring $\mathrm{CAGA}_{12}$-luciferase activity, thus testing whether these lncRNAs affected TGF $\beta$ signaling [12]. The $\mathrm{CAGA}_{12}$-luciferase reporter monitors TGF $\beta$ signaling quantitatively by recruiting to its multimeric promoter the SMAD2/SMAD3/SMAD4 complex inducing the synthesis of luciferase transcripts.

MIRIOOHG IncRNA expression was upregulated by TGF $\beta$ stimulation with a peak of induction at $24 \mathrm{~h}$ (Fig. 1c), whereas its downregulation decreased $\mathrm{CAGA}_{12}$-luciferase reporter responsiveness (see "Results") [12]. In addition to normal immortalized cells (Fig. 1c), TGF $\beta$ induced MIR100HG expression with cell-type-specific kinetics in A549 lung adenocarcinoma, PC3U prostate carcinoma, and HuCCT1 cholangiocarcinoma cells (Fig. 1d-f), and in pancreatic and lung adenocarcinoma cell line cohorts of the transcriptomic datasets GSE23952 [13] and GSE114761 [14] (Supplementary Fig. S1). These data agree with the first report of MIRIOOHG regulation by TGF $\beta$ in pancreatic tumors [6].
MIRIOOHG spans 4 exons and 3 introns (NR_024430.2 transcript) on chromosome 11q24.1 and generates a 3129-ntlong spliced RNA (Fig. 2a). Intron-3 gives rise to $M I R-100$, a precursor to mature $m i R-100-5 p /-3 p, M I R L E T 7 A 2$, generating let-7a-5p/let-7a-2-3p, MIR125B1, a precursor to mature $m i R-125 b-5 p / m i R-125 b-1-3 p$, and the BLID (BH3like motif-containing cell death inducer) protein (Fig. 2a). Chemical inhibition of TGF $\beta$ receptor I (TGF $\beta$ RI) kinase activity (TßRi: GW6604) in HaCaT cells, normalized TGF $\beta$-induced MIR100HG expression to basal levels (Fig. 2b), as well as the established TGF $\beta$-induced protein PAI-1 (plasminogen activator inhibitor-1, Fig. 2c). Silencing SMAD2, SMAD3, or SMAD4 alone or in combinations decreased TGF $\beta$-induced MIRIOOHG expression in HaCaT, PC3U, A549, and HuCCT1 cells (Fig. 2d-g and Supplementary Fig. S2a-c). In the HuCCT1 model, an independent T $\beta$ Ri (LY2157299) blocked the induction of MIRIOOHG by TGF $\beta$ (Supplementary Fig. S2d). Chromatin immunoprecipitation (ChIP) revealed the association of SMAD2/3 to the MIR10OHG promoter in $\mathrm{HaCaT}, \mathrm{PC} 3 \mathrm{U}$, and A549 cells after stimulation with TGF $\beta$ (Supplementary Fig. S2e, f).

$\mathrm{HaCaT}$ and $\mathrm{PC} 3 \mathrm{U}$ cell fractionation analysis confirmed that $>90 \%$ of $M I R 100 H G$ was cytoplasmic and $<10 \%$ was nuclear (Fig. 2h-k). HPRT1 mRNA was essentially cytoplasmic, as expected, and IncRNA TGFB2-AS1 [12] was exclusively nuclear. Fraction purity was determined by analyzing the nuclear envelope protein lamin-B1 and the cytosolic proteins GAPDH and $\alpha$-tubulin (Fig. 2i, k). Thus, several TGFß-responsive cell models exhibit TGF/RI- and SMAD2/ 3/4-dependent transcriptional induction of $M I R I O O H G$, whose spliced product accumulates in the cytoplasm. In the following experiments, we studied MIRIOOHG expression and functional roles in $\mathrm{PC} 3 \mathrm{U}, \mathrm{A} 549$, and in nontumorigenic $\mathrm{HaCaT}$ cells, as these cell models showed robust responses of MIRIOOHG to TGF $\beta$ (Fig. 1), and represent cells of independent tissue origin.

\section{MIR100HG regulates diverse cell responses to TGF $\beta$}

We investigated whether MIRIOOHG could affect expression of TGF $\beta$ target genes. Using a pool-of-4 siRNAs, two individual siRNAs targeting exons 1 and 4, and an independent antisense oligonucleotide (ASO) specific for exon 4 of $M I R 100 H G$ (Fig. 2a), we identified those that silenced MIRIOOHG in HaCaT cells (Supplementary Fig. S3a), and chose siRNA\#9 (exon 1) and ASO (exon 4) for the majority of subsequent experiments. Upon MIRIOOHG knockdown with siRNA\#9 (Fig. 3a), inducibility of SERPINE1 (encoding PAI-1), FIBRONECTIN 1 (FNI), and SNAII/SNAIL expression by TGF $\beta$ in PC3U cells was almost completely lost (Fig. 3b-d) (note retained weak inducibility of FN1, Fig. 3c), and basal SNAII expression was suppressed (Fig. 3d), consistent with a role for MIRIOOHG to promote autocrine TGF $\beta$ 
Fig. 1 MIR100HG is induced by TGF及. a Schematic outline of the experimental design to identify TGF $\beta$-regulated lncRNAs. b Total numbers of lncRNA genes regulated by

TGF $\beta 1$ in a time-course experiment, identified by microarray analysis. c-f Realtime RT-qPCR for determination of $M I R 100 H G$ expression in HaCaT (c), A549 (d), PC3U (e), and HuCCT1 (f) cells in response to TGF $\beta 1$ treatment for the indicated time periods. Gene expression is normalized relative to the housekeeping genes HPRT1 (c-e) or TBP (f). Error bars represent standard deviation from three different experiments $(* P<0.05, * * P<0.01, * * * P<$ 0.001 ). a
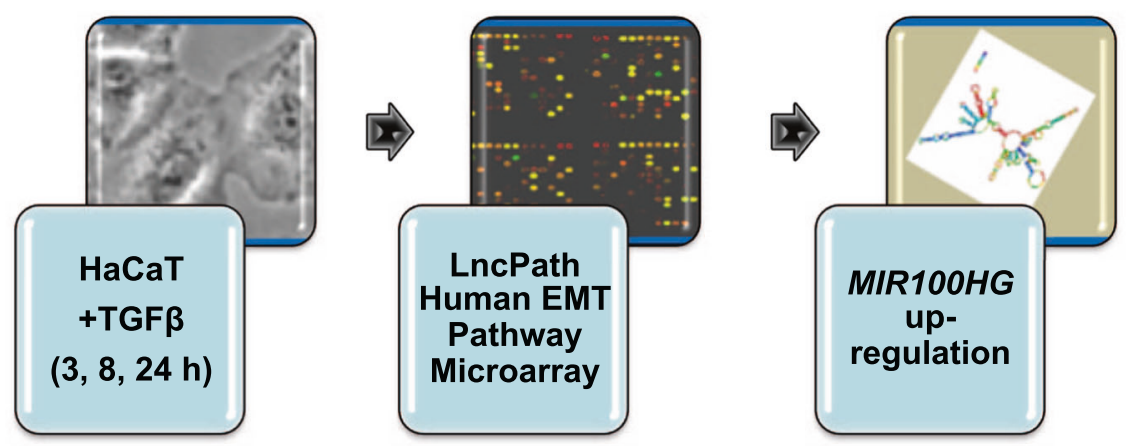

b HaCaT

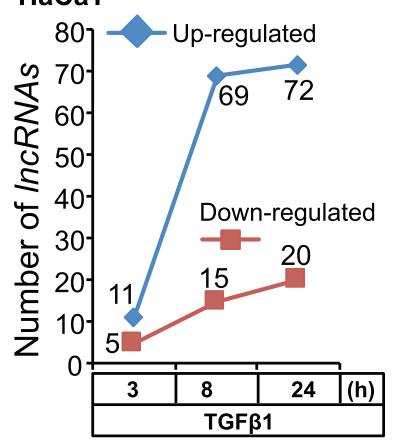

C

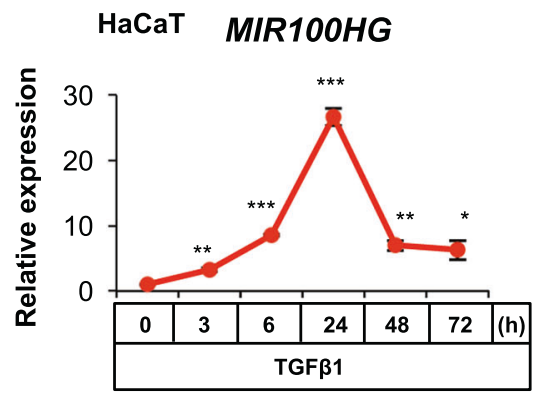

e

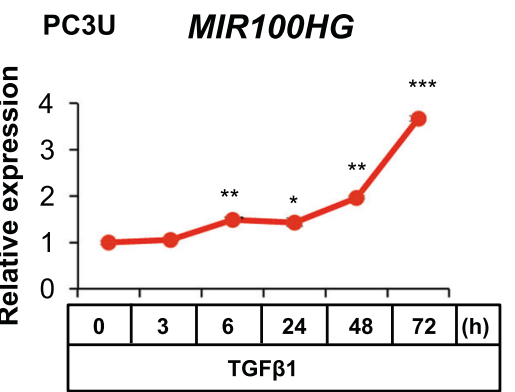

d $\quad$ A549

MIR100HG

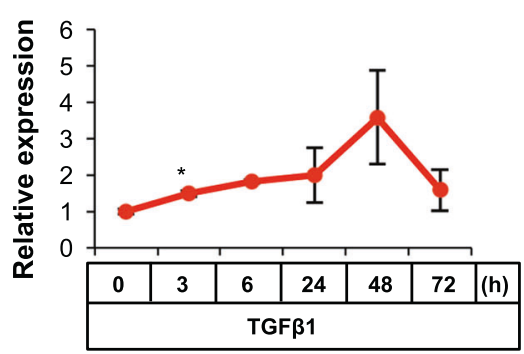

f

HuCcT1 MIR100HG

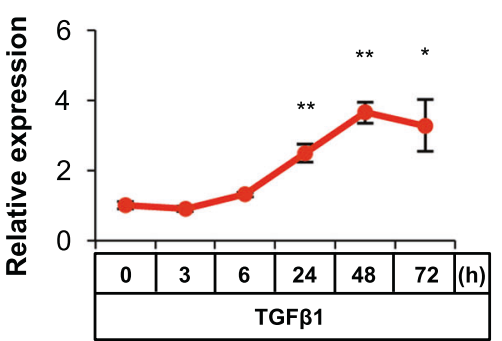

signaling. MIR100HG silencing by siRNA\#9 (Fig. 3a) reduced FN1 and PAI-1 protein levels (Fig. 3e). Although PC3U carcinoma cells do not exhibit physiological cell cycle arrest in response to TGF $\beta$, upon $M I R 100 H G$ silencing with ASO (Fig. 3f), their viability was reduced (Fig. 3g). Motility was strongly reduced in PC3U cells (Fig. 3h) upon MIR100HG silencing via ASO (Fig. 3f).
In nontumorigenic keratinocytes, MIR $100 H G$ silencing with siRNAPool-of-4 (Supplementary Fig. S3b) reduced induction of SERPINE1 and FN1 mRNAs (Supplementary Fig. S3c, d), and reduced FN1 and N-CAD protein levels, as well as phosphorylation of SMAD2 (Supplementary Fig. $\mathrm{S} 3 \mathrm{e}$ ), in response to $\mathrm{TGF} \beta$ stimulation. In these experiments, silencing of SMAD3, SMAD4 (Fig. 2e), or TGFBR2 


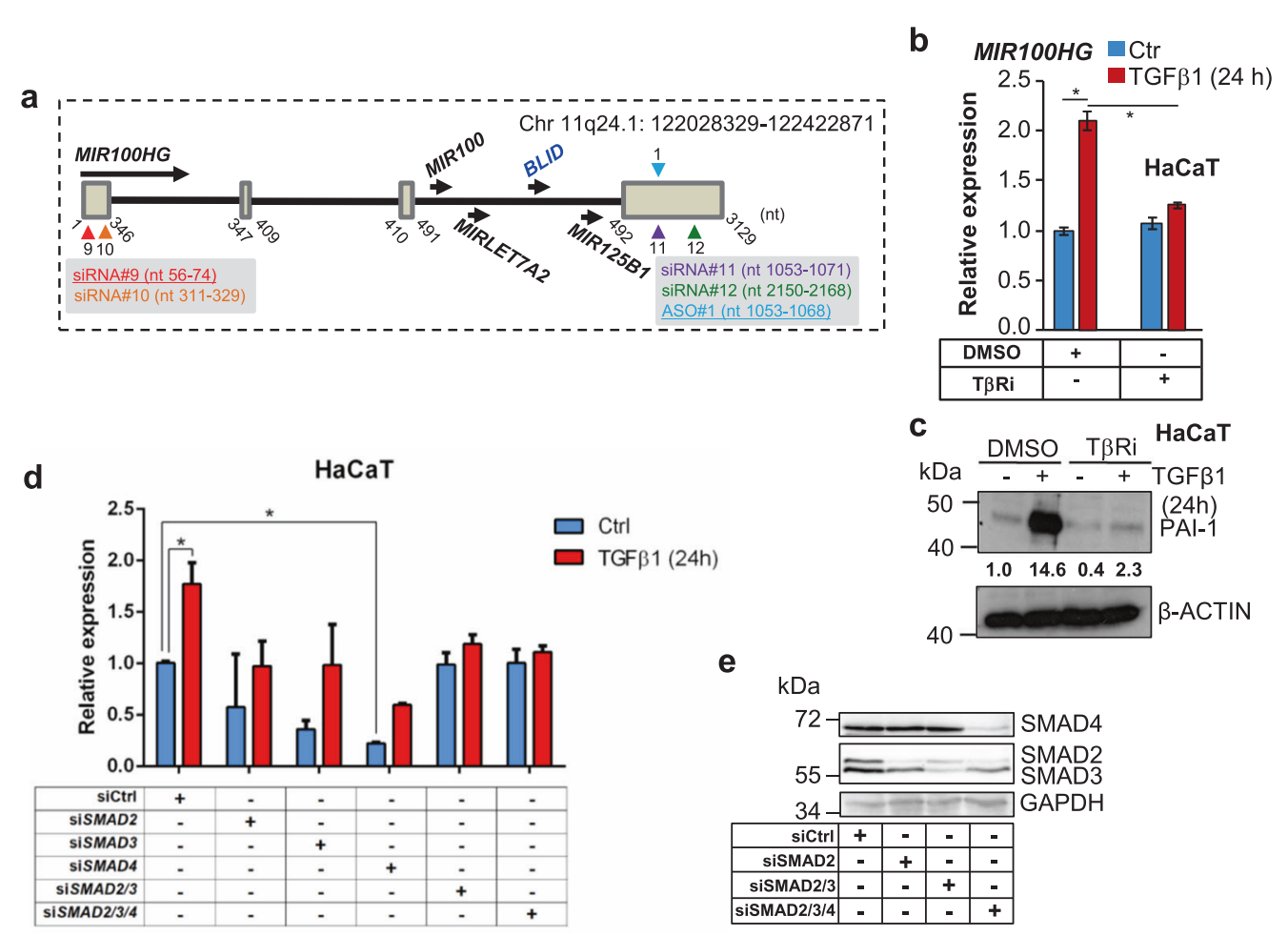

f
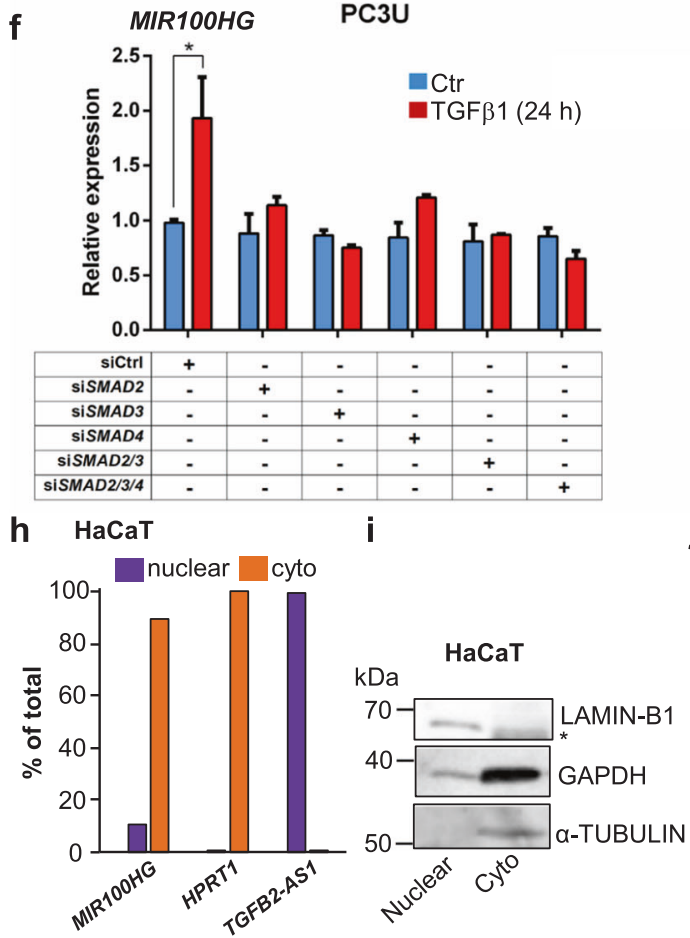

g
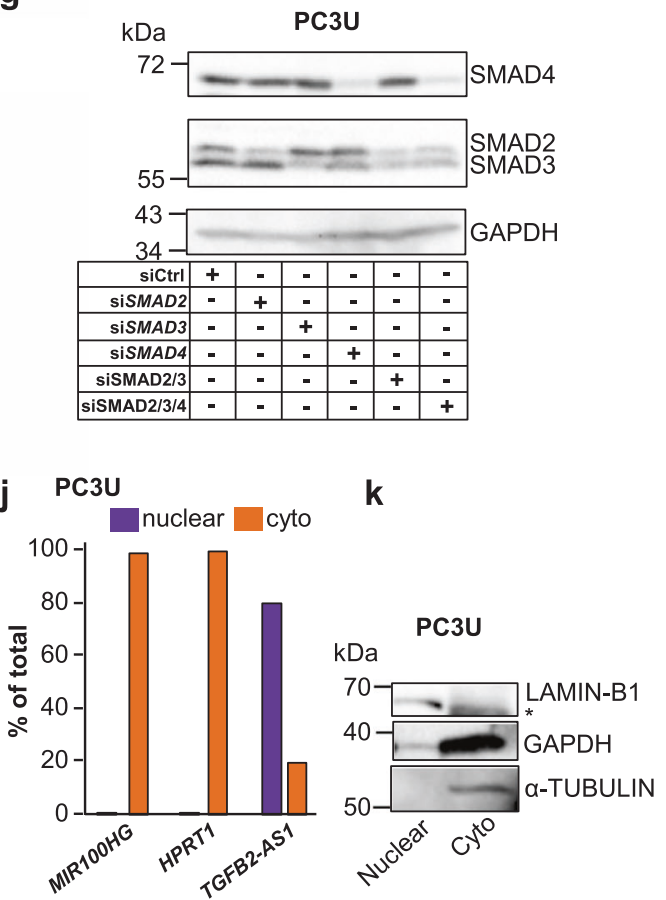

(not shown) dramatically blocked TGF $\beta$-inducible SERPINE1 and FN1 expression (Supplementary Fig. S3c, d), indicating that the impact of MIRIOOHG silencing is in the same direction but not as robust as the impact of silencing central TGF $\beta$ signaling components (TGFBR2, SMAD3, and SMAD4). TGF $\beta$-induced cell cycle arrest and cell number decrease were partially blocked (Supplementary Fig. S3e, f) after silencing $M I R 100 H G$ with siRNA\#9 or ASO (Supplementary Fig. S3a, d). Both assays also revealed a small but reproducible increase in HaCaT Sphase entry and viability upon MIRIOOHG silencing under basal conditions (Supplementary Fig. S3f, g). 
4 Fig. 2 MIR100HG is induced by TGFßRI-SMAD signaling. a Schematic representation of the organization of the MIR10OHG gene. Exons are shown as boxes and introns as lines. Arrows indicate the direction of transcription and the corresponding RNA (black) or protein-coding (blue) transcripts. The MIR100HG transcriptional unit coordinates on the $\mathrm{H} 19$ genome sequence (chromosome 11) are shown along with nucleotide (nt) numbering and coordinates of the four siRNAs and one ASO used in the study, marked by colored and numbered arrowheads, with underlines indicating those used in most experiments. b Real-time RT-qPCR for MIR100HG in HaCaT cells treated with T $\beta$ Ri (GW6604) in combination with TGF $\beta 1$ stimulation. Gene expression is normalized relative to the housekeeping gene HPRT1. Error bars represent standard deviation from three different experiments $(* P<0.05)$. c Immunoblotting for PAI-1 in HaCaT cells treated with T $\beta \mathrm{Ri}$ (GW6604) in combination with TGF $\beta 1$ stimulation for $24 \mathrm{~h}$. $\beta$-ACTIN was used as a loading control. Molecular mass $(\mathrm{kDa})$ markers are indicated along with densitometric values of normalized band intensity. d, f Real-time RT-qPCR for determination of $M I R 100 H G$ in HaCaT (d) or PC3U (f) cells transiently transfected with siRNAs targeting SMAD2, SMAD3, SMAD4, or combinations and treated or not with TGF $\beta 1$ for $24 \mathrm{~h}$. Gene expression is normalized relative to the housekeeping gene HPRT1. Error bars represent standard deviation from three different experiments $\left({ }^{*} P<0.05\right.$, $* * P<$ $0.01, * * * P<0.001)$. e, $\mathbf{g}$ Immunoblots corresponding to the experiment of panels $(\mathbf{d}, \mathbf{f})$ indicating the efficiency of SMAD protein silencing in specific combinations that cover all three SMADs examined. GAPDH was used as a loading control and molecular mass ( $\mathrm{kDa})$ markers are indicated. $\mathbf{h}-\mathbf{k}$ Expression levels of $M I R 100 H G$ lncRNA, HPRT1 mRNA, and TGFB2-AS1 lncRNA (which is known to be primarily nuclear) in nuclear and cytoplasmic fractions of $\mathrm{HaCaT}(\mathbf{h})$ or PC3U (j) lysates. Gene expression is normalized relative to the housekeeping gene HPRT1. These data show a representative experiment out of two. Corresponding immunoblot controls (i, k) verify the relative purity of cell fractions based on the nuclear (LAMIN-B1) and two cytoplasmic (GAPDH, $\alpha$-TUBULIN) protein markers. Molecular mass (kDa) markers are indicated. A star indicates a nonspecific protein band recognized by the antibody.

Motility was induced in $\mathrm{HaCaT}$ cells (Supplementary Fig. S3h) upon MIR100HG silencing via ASO (Supplementary Fig. S3a).

We cloned the human MIR100HG cDNA from immortalized mammary epithelial MCF10A cells (Supplementary Fig. S4a), and verified overexpression of MIR100HG in HaCaT (Supplementary Fig. S4b) and PC3U cells (Fig. 3i), which further enhanced basal and TGF $\beta$-induced SERPINEI, FNI, and SNAII mRNA levels and FN1 and PAI-1 protein levels in the PC3U cells (Fig. $3 \mathrm{j}-\mathrm{m}$ ), and the same proteins in $\mathrm{HaCaT}$ cells (Supplementary Fig. S4c). MIR $100 H G$ gain-of-function reduced viability of keratinocytes and cooperated with TGF $\beta$ by inducing a more robust growth inhibition (Supplementary Fig. S4d), whereas in PC3U cells, MIR100HG enhanced viability (Fig. 3n). Motility assays did not reveal significant differences upon MIR100HG overexpression in either cell model (Fig. 3o and Supplementary Fig. S4e). We conclude that MIR100HG silencing diminished, whereas MIR100HG overexpression strengthened TGF $\beta$-mediated cell responses.

\section{Impact of MIR100HG on SMAD signaling and cytotoxicity}

We investigated whether MIRIOOHG regulates basal TGF $\beta$ signaling. Similar to HaCaT cells (Supplementary Fig. S3e), silencing MIR100HG with siRNA\#9 in PC3U cells (Fig. 3a) decreased the well-established early phosphorylation of SMAD2, SMAD3, and p38 MAPK after $30 \mathrm{~min}$ of TGF $\beta$ stimulation (Supplementary Fig. S5a). Relative to the control, nonspecific ASO, which allowed proper timedependent TGF $\beta$ signaling, anti-MIRIOOHG ASO (silencing efficiency shown in Fig. 3f), decreased the phosphorylated levels of SMAD2, SMAD3, and p38 after 30 and 60 min of TGF $\beta$ stimulation (Supplementary Fig. S5b). Conversely, MIR100HG overexpression (Fig. 3i) enhanced phosphorylation of SMAD2 and SMAD3 (Fig. 4a). SMAD2/SMAD3 signaling directly translates to target gene promoter/enhancer binding and regulation. Accordingly, MIR100HG overexpression approximately doubled SMAD2/3 recruitment to the SERPINE1 promoter after TGF $\beta$ stimulation, determined by ChIP-qPCR in PC3U cells (Fig. 4b), and mirroring the impact MIR100HG overexpression has on $\mathrm{CAGA}_{12}$-luciferase activity (Fig. $4 \mathrm{c}$ ). In a similar fashion, TGF $\beta$-induced $\mathrm{CAGA}_{12}$-luciferase activity in keratinocytes was reduced upon MIR100HG silencing with the siRNApool to an extent comparable to TGFBR2 silencing (Supplementary Fig. S5c), and was further enhanced upon MIR100HG overexpression (Supplementary Fig. S5d). To explore deeper effects of MIR100HG on cell viability, we used the cytotoxic drugs doxorubicin, taxol, and 5-fluorouracil in combination with TGF $\beta$ stimulation and/or MIR100HG silencing (Fig. 4d). Drug cotreatment with TGF $\beta$ enhanced cytotoxicity only in the case of 5-fluorouracil; MIR100HG silencing reproducibly enhanced cytotoxicity by all drugs, as revealed by viability, cleaved PARP-1, and caspase-3 analysis (Fig. 4d). Thus, MIR100HG possibly acts at the level of TGF $\beta$ ligand and/or TGF $\beta$ receptors.

\section{MIR100HG regulates TGF $\beta 1$ expression via the RNA- binding protein HuR}

We examined the impact of $M I R 100 H G$ on expression of TGF $\beta$ family signaling genes using a microarray platform that provided strong indications but did not allow reproducibility assays for technical reasons (Fig. 5a). Many of the 84 investigated transcripts were readily expressed in PC3U cells (Supplementary Fig. S6). The ligands TGFB1 and $I N H B A$, their downstream effector of cell adhesion and secreted glycoprotein $T G F B I$, and downstream transcription factors $I D 1, M Y C, J U N$, and $J U N B$, were downregulated upon MIRI0OHG silencing (siRNA\#9, Fig. 3a) and upregulated upon MIR100HG overexpression (Fig. 5b, single 
a

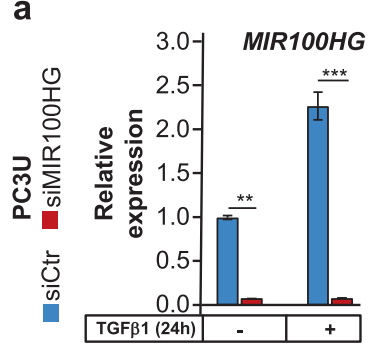

b

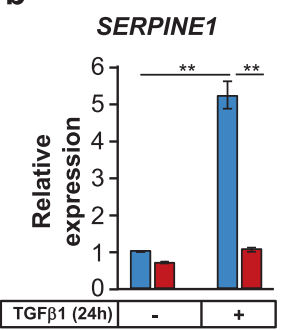

c

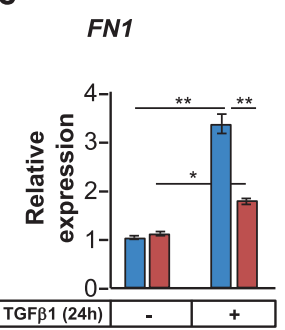

d

SNAI1 e



f

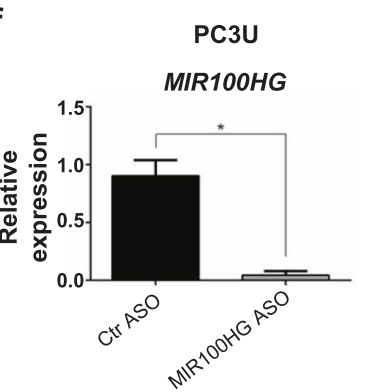

i


n

PC3U

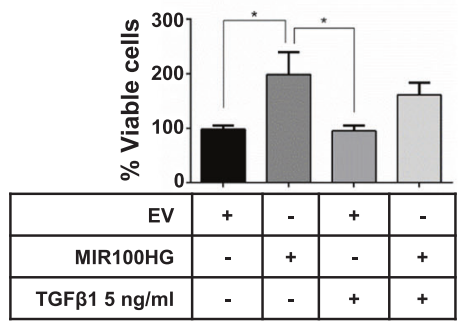

j

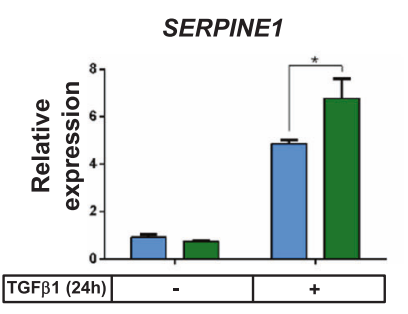

I



$\circ$

PC3U

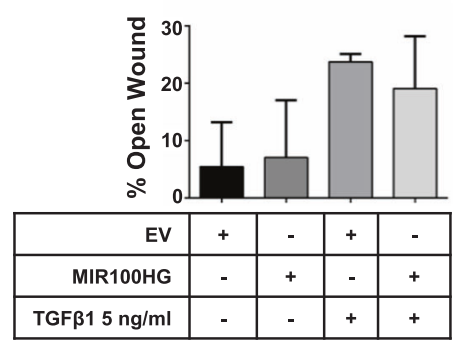

g

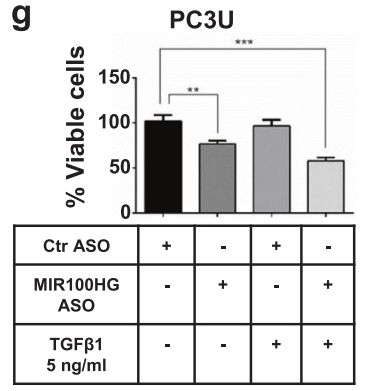

h

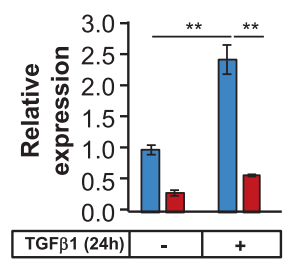

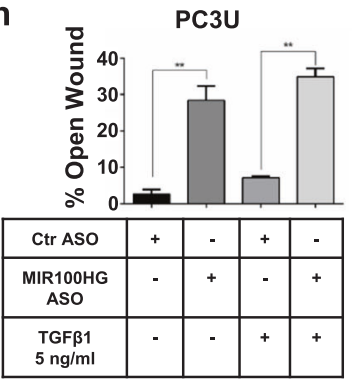

m $\quad \mathrm{PC} 3 \mathrm{U}$

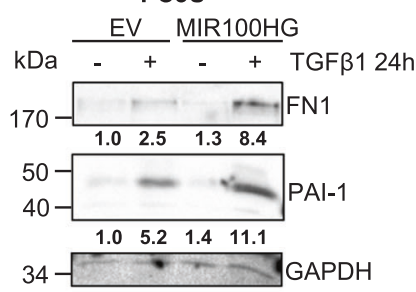

biological repeat). After TGF $\beta$ stimulation, TGFBl, $I N H B A$, and its downstream target genes IGFBP3, SERPINE1, SOX 4, THBS1, ID1, and TGFBI were most robustly inhibited after silencing $M I R 100 H G$ (Fig. 5c, single biological repeat, siRNA\#9). Independent RT-qPCR assays confirmed in multiple repeats that $M I R 100 H G$ silencing (Fig. 5d, via ASO) resulted in a relative decrease of $T G F B 1$ (see next section), BMPR2, SERPINE1, SOX4, THBS1, and TGFBI; expression of GDF2 or IDI also decreased, but not significantly (Fig. 5e), in agreement with lack of significant regulation by $M I R 100 H G$ in the single-microarray assay (Fig. 5c). Many of the analyzed genes mediate a conserved $T G F B 1$ autoinduction mechanism that responds to TGF $\beta$ signaling. 
Fig. 3 MIR100HG regulates TGF $\beta$-signaling responses. a-d Realtime RT-qPCR for detection of MIR100HG (a), SERPINE1 (b), FN1 (c), and SNAII (d) expression in PC3U cells transiently transfected with siMIR100HG\#9 and stimulated with TGF $\beta 1$ or not for $24 \mathrm{~h}$. Gene expression is normalized relative to the housekeeping gene HPRT1. Error bars represent standard deviation from three different experiments $\quad(* P<0.05, \quad * * P<0.01, \quad * * * P<0.001)$. e Representative immunoblot out of three independent experiments for FN1 and PAI-1 in PC3U cells transiently transfected with control siRNA (siC) or siMIR100HG\#9 and treated with TGF $\beta 1$ for $24 \mathrm{~h}$. $\beta$-ACTIN was used as a loading control and molecular mass $(\mathrm{kDa})$ markers are indicated along with densitometric values of normalized band intensity. f Realtime RT-qPCR for determination of MIR100HG expression in PC3U cells transiently transfected with anti-MIR100HG. Gene expression is normalized relative to the housekeeping gene HPRT1. Error bar represents the standard deviation from three different experiments $(* P$ $<0.05)$. g Cell viability/proliferation assay with PC3U cells transiently transfected with negative control (Ctr) or specific ASO and treated or not with TGF $\beta 1$ for $24 \mathrm{~h}$. Error bars represent standard deviation from three independent experiments $(* * P<0.01, * * * P<0.001)$. h Cell migration assay with $\mathrm{PC} 3 \mathrm{U}$ cells transiently transfected with negative control (Ctr) or specific ASO and treated or not with TGF $\beta 1$ for $24 \mathrm{~h}$. Error bars represent standard deviation from three independent experiments $(* P<0.05, * * P<0.01)$. i-l Real-time RT-qPCR for detection of $M I R 100 H G$ (i), which serves as a control for the experiments of panels (m-o), SERPINE1 (j), FNI (k), and SNAII (l) expression in PC3U cells transiently transfected with empty vector (EV) or pcDNA3-MIR100HG and stimulated with TGF $\beta 1$ or not for $24 \mathrm{~h}$. Gene expression is normalized relative to the housekeeping gene HPRT1. Error bars represent standard deviation from three different experiments $(* P<0.05)$. m Representative immunoblot out of three independent experiments for expression of FN1 and PAI-1 in PC3U cells transiently transfected with empty vector (EV) or pcDNA3MIR100HG and treated with TGF $\beta 1$ for $24 \mathrm{~h}$. GAPDH was used as a loading control and molecular mass $(\mathrm{kDa})$ markers are indicated along with densitometric values of normalized band intensity. $\mathbf{n}$ Cell viability/proliferation assay with $\mathrm{PC} 3 \mathrm{U}$ cells transiently transfected with empty vector (EV) or pcDNA3-MIR100HG and treated or not with TGF $\beta 1$ for $24 \mathrm{~h}$. Error bars represent standard deviation from three independent experiments $(* P<0.05)$. o Cell migration assay with PC3U cells transiently transfected with empty vector (EV) or $p$ CDNA3-MIR100HG and treated or not with TGF $\beta 1$ for $24 \mathrm{~h}$. Error bars represent standard deviation from three independent experiments. Lack of stars indicates a lack of statistical significance.

We corroborated the impact of MIR100HG on TGF $\beta 1$ expression (Fig. 6). The anti-MIR100HG ASO suppressed TGFB1 mRNA expression in PC3U and A549 cells (Fig. 6a-d) and suppressed secreted TGF $\beta 1$ protein in A549conditioned medium (Fig. 6e). MIR $100 H G$ overexpression enhanced both TGFB1 mRNA and protein secretion in PC3U cells (Fig. 6f-h). The impact MIRIOOHG had on secreted TGF $\beta 1$ suggested post-transcriptional action.

To investigate post-transcriptional mechanisms, we focused on the RNA-binding protein human antigen $\mathrm{R}$ (HuR) $[15,16]$, a nuclear protein that shuttles to the cytoplasm, associates with AU-rich sequences at the $3^{\prime}$ untranslated region of mRNAs, including TGFB1 mRNA, causing stabilization and enhancing their translation $[17,18]$. Furthermore, MIR $100 H G$ can associate with HuR, facilitating interactions between HuR and mRNAs [19].
Stimulating or not keratinocytes and PC3U cells with TGF $\beta$ confirmed that HuR partitioned mainly in the nucleus and exhibited a substantial cytoplasmic pool (Fig. 7a). Silencing HuR using two out of four independent siRNAs (Fig. 7b, c) did not significantly affect steady-state MIRIOOHG levels, but decreased TGFB1 mRNA levels (Fig. 7d, e), and weakly but not-significantly decreased secreted TGF $\beta 1$ protein (Supplementary Fig. S7).

RNA immunoprecipitation (RIP) assays in PC3U cells transfected with control or anti-MIR100HG ASO, exhibiting robust $M I R 100 H G$ silencing and corresponding TGFBI mRNA reduction (Supplementary Fig. S8a, b), demonstrated HuR immunocomplexes with high amounts of MIRIOOHG (relative to control $\mathrm{IgG}$ ), and measurable amounts of TGFB1 mRNA (Supplementary Fig. S8c, d). Calculating fold enrichment of each RNA in HuR immunocomplexes relative to nonspecific IgG, showed that TGF $\beta$ stimulation significantly enhanced HuR-MIRIOOHG association, and MIRIOOHG silencing eliminated these complexes, as expected (Fig. 7f). Confirming our hypothesis, the HuR-TGFB1 complexes lost a large portion of bound TGFB1 mRNA upon MIR100HG silencing (Fig. $7 \mathrm{~g}$ and Supplementary Fig. S8d). As an additional control, TGFB1 was silenced, decreasing basal MIRIOOHG levels as expected (Fig. 7h), and after TGF $\beta$ stimulation, causing observable but weaker MIRIOOHG decrease (Supplementary Fig. S8e, f). RIP demonstrated again HuR-TGFBI association, which decreased, as expected, after TGFBI silencing (Fig. 7i and Supplementary Fig. S8g), and HuRMIR100HG complexes showed a decreasing but not statistically significant trend (Fig. $7 \mathrm{j}$ and Supplementary Fig. $\mathrm{S} 8 \mathrm{~h})$. The data suggest that MIR $100 H G$ facilitates the formation of HuR-TGFB1 ribonucleoprotein complexes (Fig. 7k).

\section{Regulation of miRNA expression from the MIR100HG intron-3}

Since unspliced MIR100HG can be processed into miRNAs (Fig. 2a), signaling inputs that induce spliced MIR100HG should regulate MIR100HG-derived miRNAs, as they share the same transcriptional promoter. Pre-miR100 showed time-dependent downregulation in $\mathrm{HaCaT}$ cells and stable levels in PC3U cells in response to TGF $\beta$ stimulation (Supplementary Figs. S9a, S10a). Mature $m i R-100-5 p$ showed a late-time but not significant trend of upregulation in both cell lines (Supplementary Figs. S9b, S10b), and also increased weakly in A549 cells after 24-h stimulation (Supplementary Fig. S11a). MiR100-3p expression was undetectable in all cell lines. MiR-361-5p expression, used as a reference, remained unchanged in response to TGF $\beta$ (Supplementary Fig. S11b). Pre-miR-125b showed early induction by TGF $\beta$ in 
a

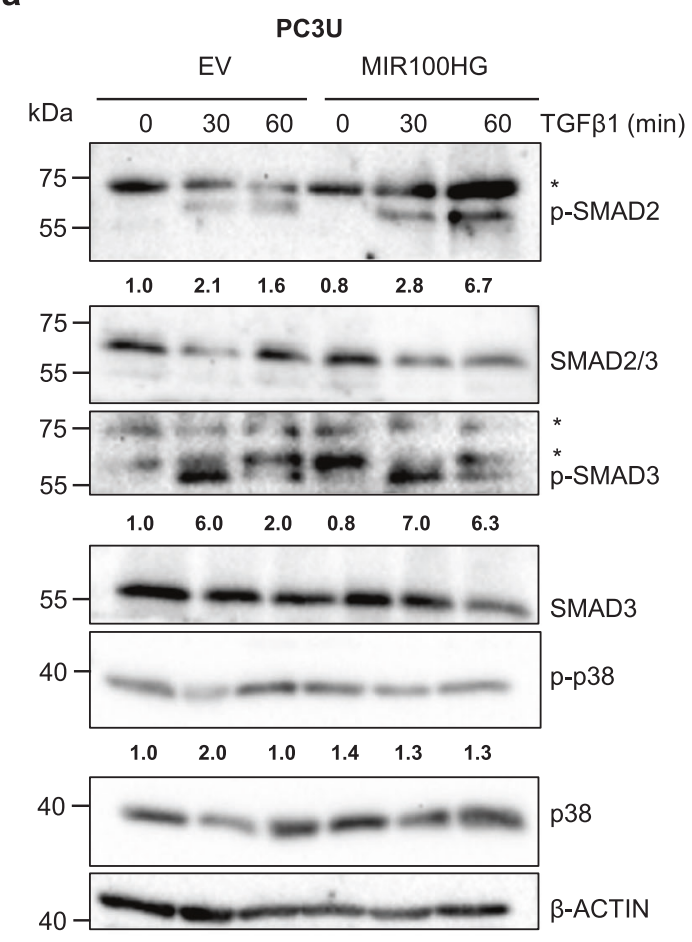

b

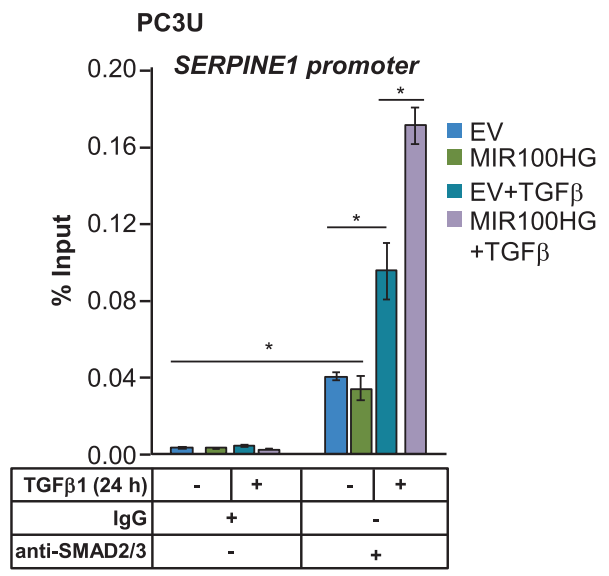

C

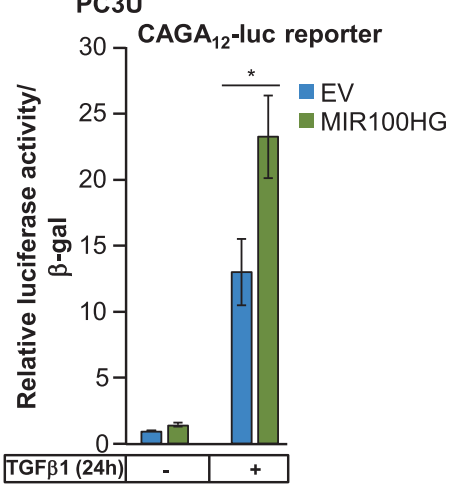

d

PC3U

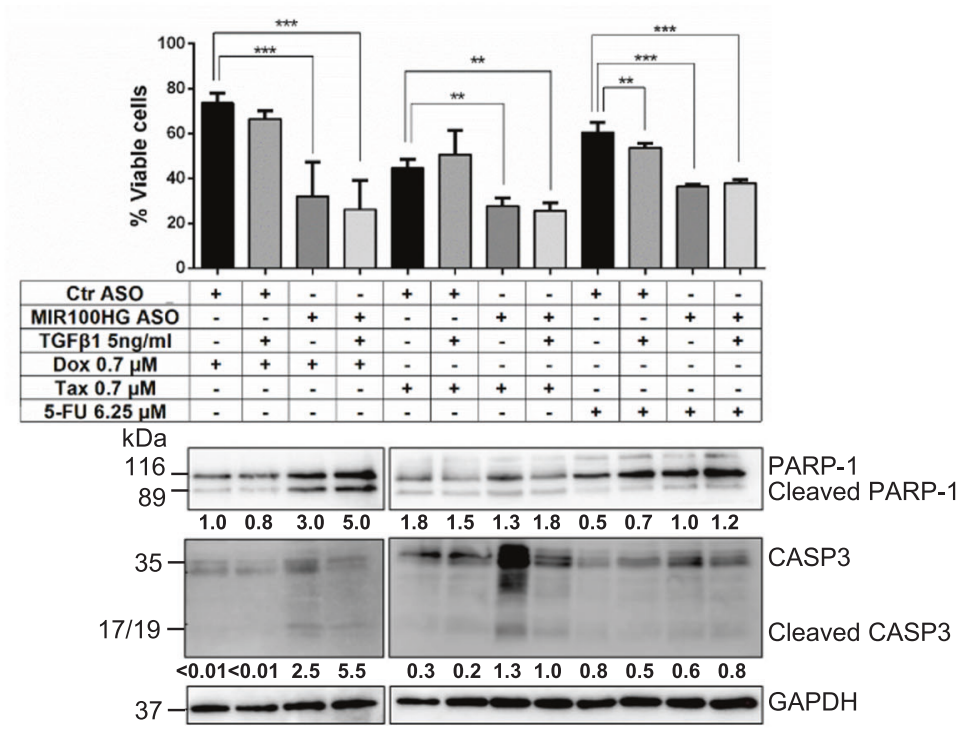

$\mathrm{HaCaT}$ cells and early downregulation in PC3U cells (Supplementary Figs. S9c, S10c). The corresponding mature miRNAs showed (not significant) trends for upregulation in HaCaT (Supplementary Fig. S9d, e), early downregulation but only for miR-125b-3p in PC3U cells (Supplementary Fig. S10d, e), and significant upregulation at $24 \mathrm{~h}$ in A549 cells (Supplementary Fig. S11c, d). PremiR-let7a-2 showed early upregulation in $\mathrm{HaCaT}$ cells and a corresponding (but not significant) trend in PC3U cells (Supplementary Figs. S9f, S10f). Mature let-7a-2 miRNAs showed corresponding trends of upregulation in $\mathrm{HaCaT}$ and PC3U cells (Supplementary Figs. S9g, h, S10g, h), 
Fig. 4 MIR100HG regulates TGF $\beta$ receptor signaling. a Representative immunoblots out of three independent experiments for expression of phosphorylated SMAD2 (p-SMAD2), SMAD2/3, phosphorylated SMAD3 (p-SMAD3), SMAD3, phosphorylated p38 (p-p38), and $\mathrm{p} 38$ in PC3U cells transiently transfected with empty vector $(E V)$ or pcDNA3-MIR100HG (as shown in Fig. 3i) and treated with TGF $\beta 1$ for the indicated time periods. $\beta$-ACTIN was used as a loading control and molecular mass $(\mathrm{kDa})$ markers are indicated along with densitometric values of normalized band intensity. Stars indicate nonspecific protein bands recognized by the antibodies. b ChIP-qPCR analysis for SMAD2/ 3 occupancy to the SERPINE1/PAI-1 promoter in PC3U cells stimulated with TGF $\beta 1$ for $24 \mathrm{~h}$ or not, after transient transfection of empty vector (EV) or pcDNA3-MIR100HG (as shown in Fig. 3i). Control IgG immunoprecipitation data are also shown. Error bars represent standard deviation from three different experiments $(* P<0.05)$. c CAGA 12 -luciferase assay in $\mathrm{PC} 3 \mathrm{U}$ cells transiently transfected with empty vector $(\mathrm{EV})$ or pcDNA3-MIR100HG and in the presence or absence of TGF $\beta 1$ stimulation for $24 \mathrm{~h}$ (as shown in Fig. 3i). Error bars represent standard deviation from three different experiments $(* P<0.05)$. d Cell viability assay with $\mathrm{PC} 3 \mathrm{U}$ cells transiently transfected with negative control (Ctr) or specific ASO (as shown in Fig. 3f) and treated with TGF $\beta 1$ in the absence or presence of the indicated concentrations of doxorubicin (Dox), taxol (Tax), or 5'-fluorouracil (5-FU) for $48 \mathrm{~h}$. Error bars represent standard deviation from three independent experiments $(* * P<0.01, * * * P<0.001)$.

whereas significant upregulation only for let-7a-2-3p was observed in A549 cells (Supplementary Fig. S11e, f). Thus, TGF $\beta$ can regulate expression of some of the miRNAs of the $M I R 100 H G$ intron-3, in a cell-type- and timedependent manner; however, the kinetics of regulation of the miRNAs do not match those of spliced MIRIOOHG and the regulation is highly variable.

In order to test whether spliced MIR10OHG affects intron3 miRNA biogenesis, we silenced spliced MIR100HG (using siRNApool, Supplementary Fig. S12a) in $\mathrm{HaCaT}$ cells, and detected no impact on basal expression of the five miRNAs or on TGF $\beta$-induced levels of miR-125b-1-3p and let-7a2-3p (Supplementary Fig. S12). In PC3U cells though, MIR100HG silencing (via ASO, Supplementary Fig. S3a) had no effect on pre-miR-100, but resulted in significant downregulation of mature miR-100-5p, pre-miR-125b, and $m i R-125 b-1-3 p / 5 p$ (Supplementary Fig. S13). The cumulative data suggest that spliced $M I R 100 H G$ functions in parallel and possibly independently from the intron-3 miRNAs, however, a clear impact on mature $m i R-100-5 p, m i R-125 b$ $5 p$, and $m i R-125 b-1-3 p$ expression is worth considering.

\section{Let-7a-2-3p regulates TGF $\beta$-target genes and epithelial cytostasis}

We also asked whether intron-3 miRNAs could affect TGF $\beta$ signaling output. We overexpressed chemically stabilized mimics of mature miRNAs whose expression was regulated by TGF $\beta$ (let-7a-2-3p, miR-125b-1-3p, and miR-125b-5p) or negative control random-sequence miRNA mimic $(\mathrm{NCm})$ in HaCaT cells. Let-7a-2-3p mimic but not miR-125b-1-3p mimic or miR-125b-5p mimic potentiated TGF $\beta$-induced CAGA $_{12}$-luciferase activity (Supplementary Fig. S14a). Cotransfecting all three miRNA mimics (let-7a-2-3p, miR$125 b-5 p$, and miR-125b-1-3p) enhanced TGF $\beta$-induced $\mathrm{CAGA}_{12}$-luciferase activity (Supplementary Fig. S14b), to a similar degree as achieved by single let-7a-2-3p mimic, failing to demonstrate additive effects.

Furthermore, ectopic let-7a-2-3p mimic, enforced significant cell cycle arrest, whereas $m i R-125 b-5 p$ mimic and $m i R-125 b-1-3 p$ mimic showed a trend but not significant effect (Supplementary Fig. S14c). TGF $\beta$ stimulation further enhanced the cytostatic response (Supplementary Fig. $\mathrm{S} 14 \mathrm{c})$. We also monitored let-7a-2-3p effects on expression of CDK inhibitors downstream of TGF $\beta$ signaling, observing enhancement of $C D K N 2 B / p 15^{\text {Ink } 4 b}$ expression induced by TGF $\beta$ stimulation, and strong upregulation of $C D K N 1 A$ / $p 21^{\text {Cipl }}$ expression in control and TGF $\beta$-treated cells (Supplementary Fig. S14d, e).

Analyzing genes of the TGF $\beta$ fibrogenic program, let-7a2-3p mimic enhanced TGF $\beta$-induced SERPINEI and FNI expression (Supplementary Fig. S14f). Inhibiting endogenous let-7a-2-3p but not let-7a-5p (produced from the $5^{\prime}$-arm of the pre-let-7a-2) or the reference gene $m i R-361-5 p$, weakly attenuated TGF $\beta$-induced SERPINE1 and FNI levels, PAI-1 and N-CAD protein levels, without clear effect on FN1 protein, and phospho-SMAD2 levels in HaCaT cells (Supplementary Fig. S15).

We attempted to identify potential targets of let-7a-2-3p using the DIANA online suite [20]. After removing 54 common predicted targets of let-7a-5p and let-7a-2-3p, we observed several highly significant pathways encompassing the target mRNAs, including glycosaminoglycan biosynthetic enzymes, Wnt signaling components, and histone methyltransferases (Supplementary Fig. S16a, b). Parallel querying of the KEGG database for all let-7a-2 target mRNAs revealed additional and diverse functional categories (Supplementary Fig. S16c-e). Focusing on the let-7a-2-3p targets, Wnt and estrogen signaling are known to crosstalk with TGF $\beta$ and provide relevant points for analyzing the role of let-7a-2 in the context of cancer biology. Collectively, intron-3 miRNAs can, to some extent, contribute to TGF $\beta$ responses, whereas the impact of MIRIOOHG was more robust and general.

\section{MIR100HG and TGF $\beta 1$ expression profiles in diverse tumors}

Since TGF $\beta$ induces $M I R 100 H G$, which enhances TGF $\beta$ signaling in normal and carcinoma cells, we examined MIR100HG expression in cancer patients. Querying the PanCancer Atlas expression data from 10,000 patients [21], revealed that $M I R 100 H G$ expression was significantly higher in the lung (where A549 cells belong) and prostate 
a

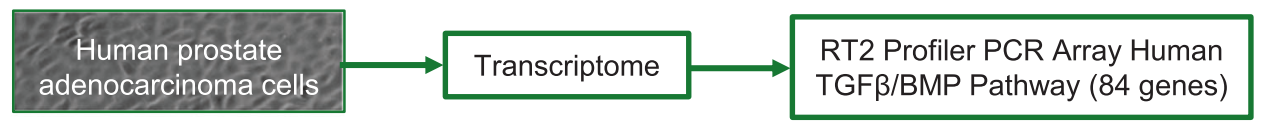

b

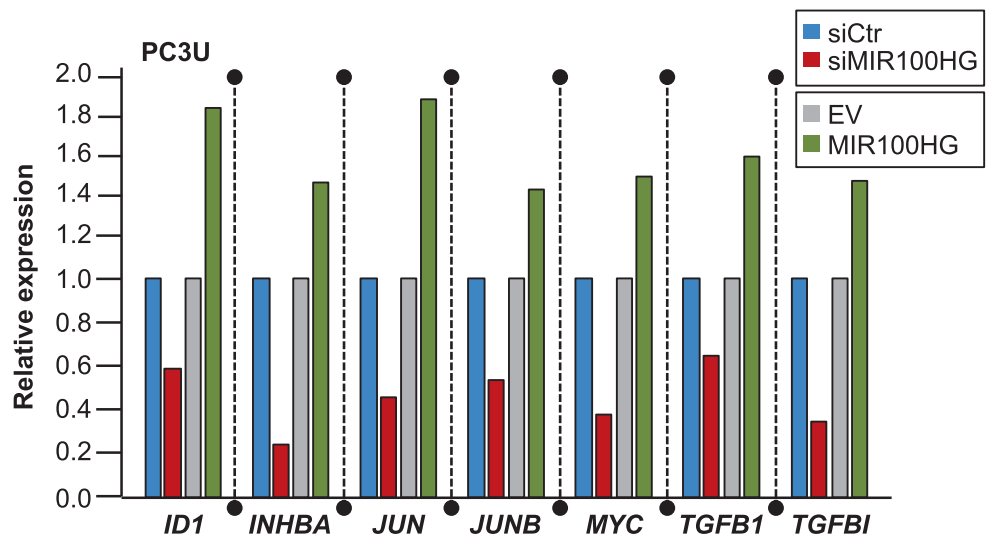

C

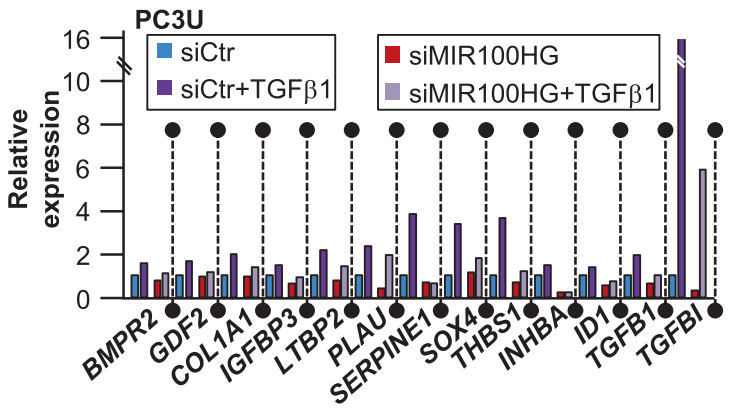

d PC3U $=\mathrm{Ctr}$ ASO $\square \mathrm{MIR} 100 \mathrm{HG}$ ASO MIR100HG

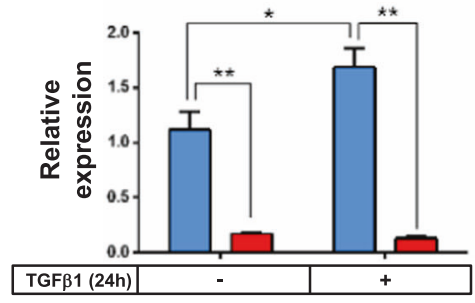

e

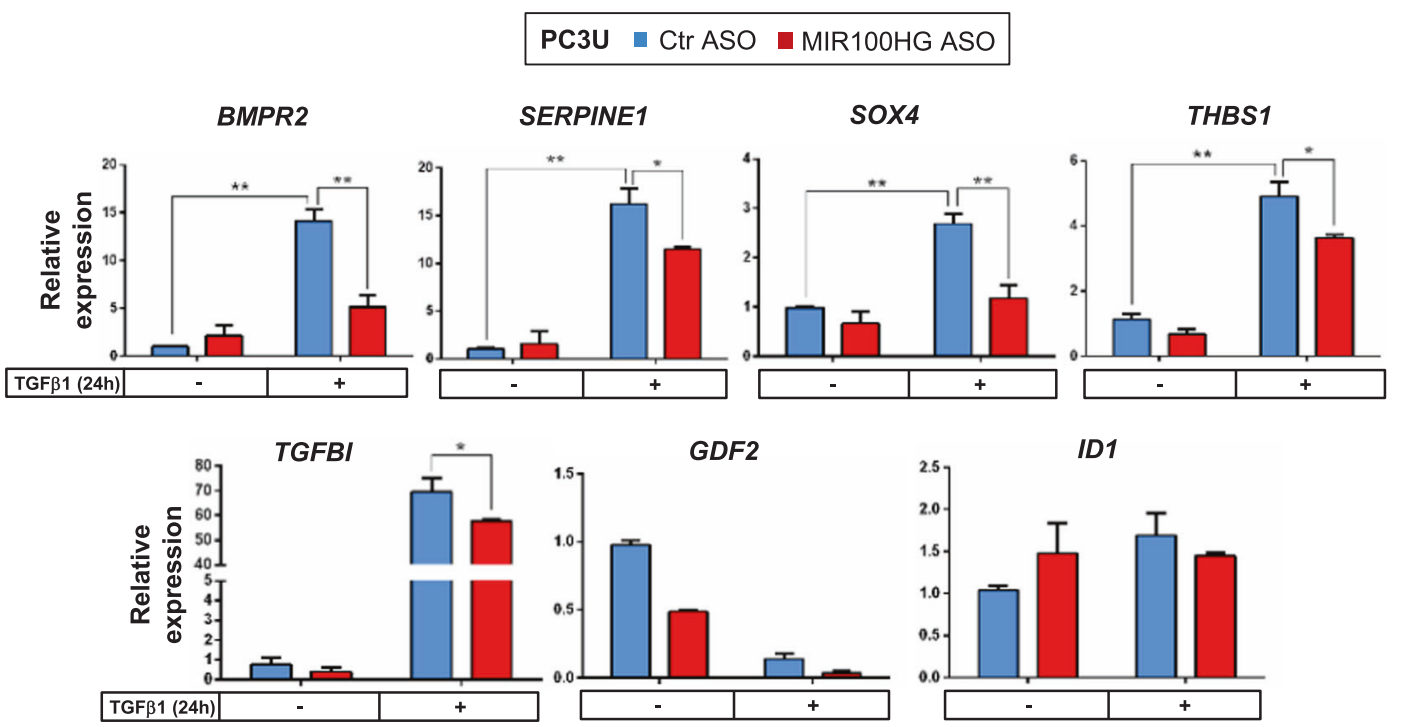

Fig. 5 MIR100HG regulates TGFB1 expression and many members of the fibrogenic program. a Schematic outline of the experimental design to identify TGF $\beta$-signaling targets of $M I R 100 H G$ action. $\mathbf{b}$ Gene expression data based on the RT ${ }^{2}$ profiler PCR array of the human TGF/BMP signaling pathway in PC3U cells transiently transfected with control siRNA (siCtrl) or siMIR100HG\#9 (as shown in Fig. 3a) and with empty vector (EV) or pcDNA3-MIR100HG (as shown in Fig. 3i). Data from a single biological repeat are shown, highlighting genes whose expression was affected by both silencing and overexpression of $M I R 100 H G$. c Similar experiment as in panel (b) with the addition of TGF $\beta 1$ stimulation for $24 \mathrm{~h}$ in PC3U cells. $\mathbf{d}$, $\mathbf{e}$ Validation of PCR array analysis using real-time RT-qPCR for detection of MIR10OHG, BMPR2, SERPINE1, SOX4, THBS1, TGFBI, $G D F 2$, and $I D 1$ expression in PC3U cells transiently transfected with negative control or specific ASO and stimulated or not with TGF $\beta 1$ for $24 \mathrm{~h}$. Gene expression is normalized relative to the housekeeping gene HPRT1. Error bars represent standard deviation from three different experiments $(* P<0.05, * * P<0.01)$. 
a

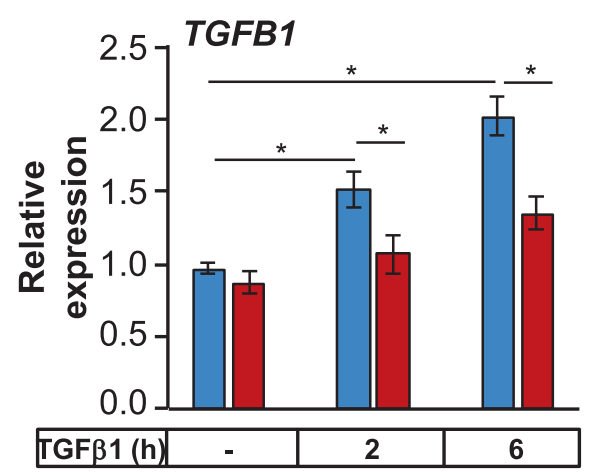

Neg Ctr ASO

MIR100HG ASO b PC3U

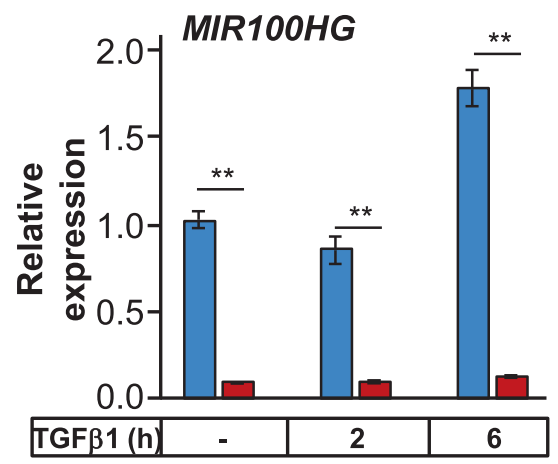

C

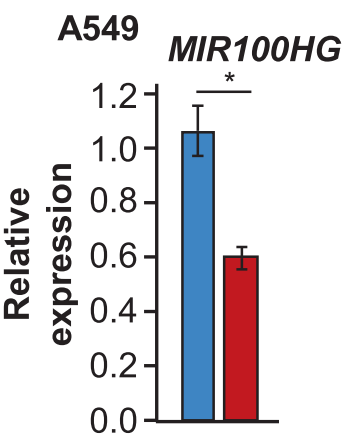

Ctr ASO

MIR100HG ASO d

A549

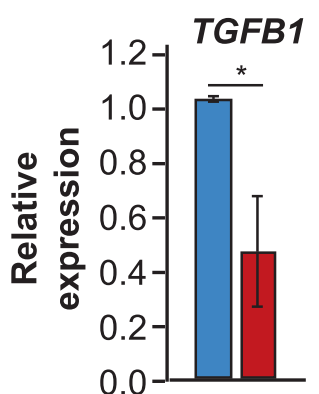

Ctr ÁSO

MIR100HG ASO

e



Z Ctr ASO

ZMIR100HG ASO
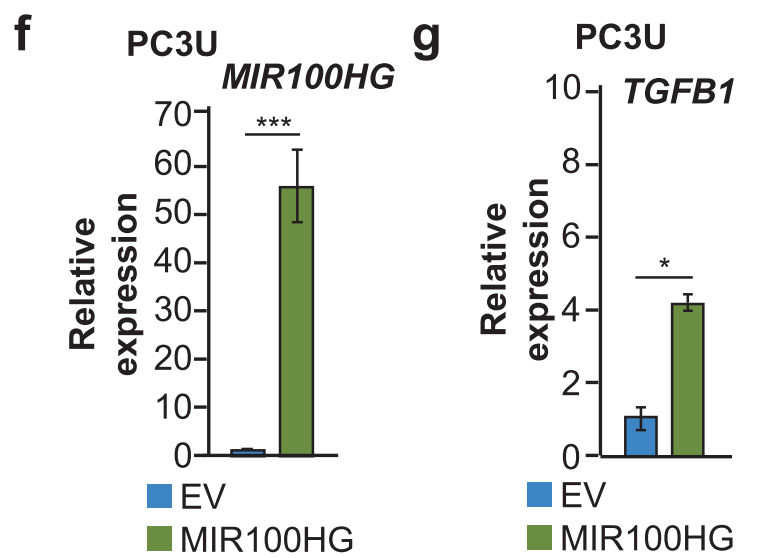

Fig. 6 TGFß1 synthesis and secretion is induced by MIR100HG downstream of TGF $\beta$ signaling. a, b Real-time RT-qPCR for detection of TGFB1 (a) and MIR100HG (b) expression in PC3U cells transiently transfected with negative control or MIR100HG-specific ASO and stimulated with TGF $\beta 1$ or not for the indicated time periods. Gene expression is normalized relative to the housekeeping gene HPRT1. Error bars represent standard deviation from three different experiments $\left({ }^{*} P<0.05,{ }^{*} P<0.01\right)$. c-h Detection of $M I R 100 H G$

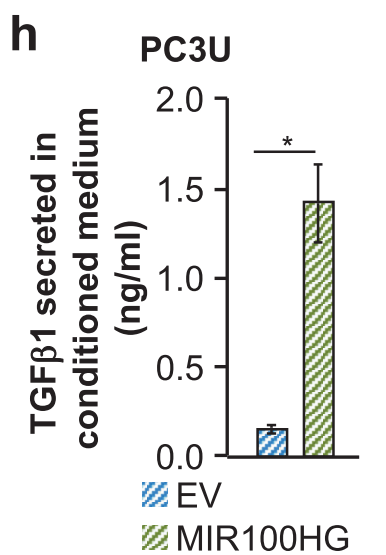

(c, f), TGFB1 mRNA (d, g) expression by real-time RT-qPCR, and corresponding secreted mature TGF $\beta 1$ ligand detected by ELISA in the conditioned medium $(\mathbf{e}, \mathbf{h})$ of A549 (c-e) and PC3U (f-h) cells transiently transfected with negative control or $M I R 100 H G$-specific ASO in the absence of TGF $\beta$ stimulation. Gene expression is normalized relative to the housekeeping gene HPRT1. Error bars represent standard deviation from three different experiments $\left({ }^{*} P<\right.$ $0.05, * * * P<0.001)$. 
a

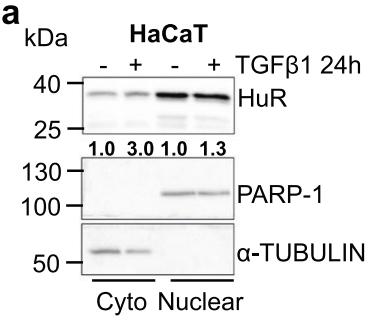

C

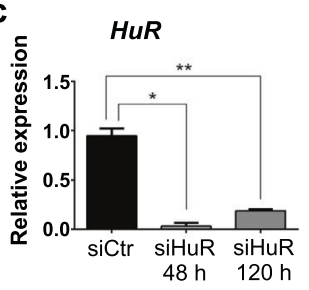

f

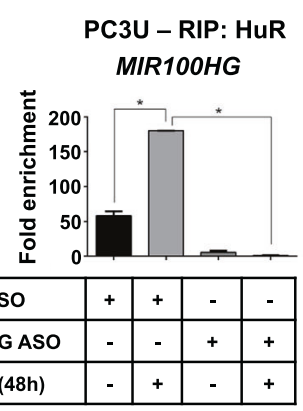

h

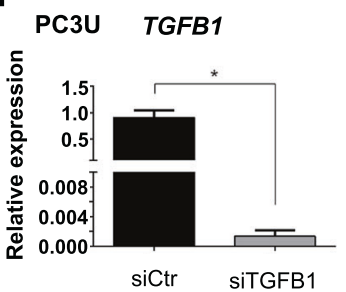

i

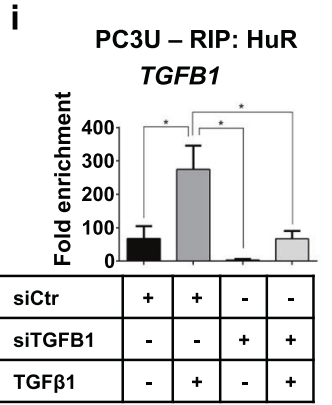

j

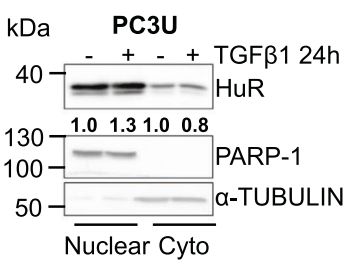

d

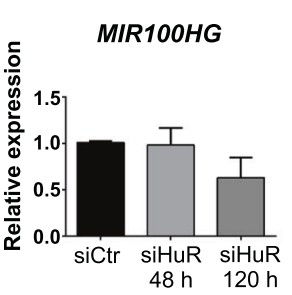

b



e



g

PC3U - RIP: HUR

TGFB1



\begin{tabular}{|c|c|c|c|c|}
\hline Ctr ASO & + & + & - & - \\
\hline MIR100HG ASO & - & - & + & + \\
\hline TGF $\beta 1(48 \mathrm{~h})$ & - & + & - & + \\
\cline { 4 - 6 }
\end{tabular} \begin{tabular}{c|c|c|c|c|c|} 
Ctr ASO & + & + & - & - \\
\hline MIR100HG ASO & - & - & + & + \\
\hline TGF $\beta 1(48 \mathrm{~h})$ & - & + & - & + \\
\hline
\end{tabular}


(where PC3U cells belong) carcinoma relative to other tumors (Supplementary Fig. S17a). A PanCancer Atlas cohort of 494 prostate adenocarcinoma patients [21] revealed a positive correlation between $M I R I 00 H G$ and $T G F B 1$ expression (Fig. 8a), and even stronger correlation between MIRIOOHG and TGFBI expression (Fig. 8b). Significant correlations with weaker coefficients were observed in 566 lung adenocarcinoma samples and in 36 cholangiocarcinoma samples of the PanCancer Atlas (Fig. 8c-e), the latter being confirmed with data from an independent cholangiocarcinoma cohort representing 29 patients (Fig. 8f). Using in situ hybridization on a cancer patient tissue microarray, we detected distinct cytoplasmic MIR100HG signals in lung adenocarcinoma, higher expression in malignant melanoma, and even higher in glioma specimen (Supplementary Fig. S17b), reflecting the 
4 Fig. 7 MIR100HG engages HuR to regulate TGFß1. a Representative immunoblots out of two independent experiments for expression of $\mathrm{HuR}$ in $\mathrm{HaCaT}$ (left) and $\mathrm{PC} 3 \mathrm{U}$ (right) cells treated with TGF $\beta 1$ for $24 \mathrm{~h}$. PARP-1 (nuclear) and $\alpha$-TUBULIN (cytoplasmic) were used as fractionation controls and molecular mass ( $\mathrm{kDa}$ ) markers are indicated along with densitometric values of normalized band intensity. b-e Representative immunoblot (b) out of three independent experiments for expression of $\mathrm{HuR}$, and corresponding real-time RTqPCR for detection of $H u R$ (c), MIR100HG (d), and TGFB1 (e) mRNA expression in PC3U cells transiently transfected with negative control or specific siRNA for the indicated time periods. GAPDH was used as loading control and molecular mass $(\mathrm{kDa})$ markers are indicated. A star indicates nonspecific protein bands recognized by the antibody. Gene expression is normalized relative to the housekeeping gene HPRT1. Error bars represent standard deviation from three different experiments $(* P<0.05, * * P<0.01$, $* * * P<0.001)$. f, g RIP analysis in PC3U cells transiently transfected with negative control or $M I R 100 H G$-specific ASO and stimulated with TGF $\beta 1$ or not for $48 \mathrm{~h}$. Fold enrichment of the HuR-specific RIP relative to the IgG control is reported for $M I R 100 H G$ (f) and TGFB1 (g) RNAs. Error bars represent standard deviation from three different experiments $(* P<0.05)$. h Real-time RT-qPCR for detection of TGFB1 and MIR100HG expression in PC3U cells transiently transfected with negative control or TGFB1-specific siRNA and treated with TGF $\beta 1$ for $48 \mathrm{~h}$. Gene expression is normalized relative to the housekeeping gene HPRT1. Error bars represent standard deviation from three different experiments $(* P<0.05)$. $\mathbf{i}, \mathbf{j}$ RIP analysis in PC3U cells transiently transfected with negative control or TGFB1-specific siRNA and stimulated with TGF $\beta 1$ or not for $48 \mathrm{~h}$. Fold enrichment of the HuR-specific RIP relative to the $\operatorname{IgG}$ control is reported for TGFB1 (i) and MIR100HG (j) RNAs. Error bars represent standard deviation from three different experiments $(* P<0.05)$. $\mathbf{k}$ Diagrammatic scheme of TGF $\beta$ signaling regulating the downstream target genes MIR100HG and TGFB1, mediated by the two TGF $\beta$ receptor kinases and SMAD complexes together with gene-specific transcription factors (TF, note color differentiation based on gene specificity), leading to transcriptional induction of MIR100HG and TGFB1. Two possible (dashed red arrows) and one confirmed mechanism of action of $M I R I O 0 H G$ are illustrated: (a) cytoplasmic MIR $100 H G$ associates with HuR in the cytoplasm (HuR domains are highlighted) and causes stabilization and accumulation of TGFB1 mRNA, which leads to enhanced synthesis of latent and mature secreted TGF $\beta 1$ (dimeric circles-mature TGF $\beta 1$ with twinkled lines- $\mathrm{N}$-terminal pro-domain) that further stimulates the pathway in an autocrine manner. The two RNAs are shown to interact with distinct HuR RRM domains (not proven) and for completion, nuclear HuR is also illustrated. (b) Cytoplasmic MIR10OHG promotes or stabilizes TGF $\beta$ receptor-SMAD complexes that prolong signaling. (c) A transcriptional mechanism in which nuclear MIR100HG enhances SMAD-mediated transcription of the $T G F B 1$ gene.

findings of the PanCancer Atlas cohorts (Supplementary Fig. S17a).

Since TGF $\beta$ signaling is known to play both tumor suppressor and protumorigenic roles dependent on the cancer type and stage [9], we analyzed contributions of MIR100HG and TGFB1 to overall survival of patients with different tumors. Thus, using OncoLnc [22], Kaplan-Meier analysis revealed that in lung adenocarcinoma and cutaneous melanoma, high expression of both MIRIOOHG and TGFBI was linked to long patient survival (Supplementary Fig. S18a-d), suggesting their tumor-suppressive role. Conversely, high $M I R 100 H G$ and $T G F B 1$ expression correlated with poor survival outcome in stomach adenocarcinoma (Supplementary Fig. S18e, f). The cancer database analyses must be considered with caution, yet they suggest that MIRIO0HG and TGFBI present similar predictive values in the prognosis of certain cancers.

\section{Discussion}

We here establish that TGF $\beta$ upregulates spliced $M I R 100 H G$, which positively regulates TGF $\beta$ responses in several normal and cancer cell types (Figs. 1-4). Signaling via the TGF $\beta$ RI and SMAD proteins (Fig. 2), but possibly additional mediators (e.g. MAPK) participate in this regulation, as previously established for pancreatic carcinomas [6]. Mechanistically, MIR100HG facilitates the RNAbinding protein HuR to form complexes with the TGFBI mRNA, thus stabilizing the mRNA and enhancing autocrine TGF $\beta 1$ production and autogenous responses (Fig. 7k). Our observations are compatible with recent findings that demonstrated a function of $M I R 100 H G$ as a HuR protein facilitator [19], and extend this model in the context of TGF $\beta$ cancer biology. The $3^{\prime}$ untranslated region of the TGFB1 mRNA contains AU-rich sequences recognized by HuR [18]. Furthermore, HuR-mediated stabilization of TGFB1 mRNA can sustain TGF $\beta$ signaling in cardiac fibroblasts during fibrosis [17]. Combined RIP and cell fractionation analyses confirm this model in the context of cancer cell biology (Fig. 7). In fibroblasts, TGF $\beta$ signaling was shown to induce translocation of nuclear HuR to the cytoplasm where association with mRNA seems to take place [17]. In normal epithelial and carcinoma cells, we observed weak mobilization of nuclear HuR to the cytoplasm in response to TGF $\beta$ (Fig. 7a). Yet, we find more convincing the fact that MIRIOOHG accumulates in the cytoplasm of all cell types examined (Fig. 2h-k), suggesting that TGFB1 mRNA stabilization depends on cytoplasmic accumulation of MIRIOOHG. Interestingly, RIP experiments reproducibly showed that TGF $\beta$ signaling enhanced association between HuR, $M I R 100 H G$, and TGFB1 mRNA (Fig. $7 \mathrm{~g}-\mathrm{i}$ ), suggesting that TGF $\beta$, in addition to promoting transcription from the $M I R 100 H G$ and TGFB1 genes, also regulates cytoplasmic RNA-protein assembly (Fig. 7k). HuR encompasses three RNA-recognition motifs (RRM1-3, Fig. 7k), one of which facilitates HuR dimerization [23]. We envision a mechanism whereby MIR $100 H G$ binding to one RRM, facilitates the association of TGFB1 mRNA with a second RRM in dimeric HuR (Fig. 7k).

Beyond the above mechanism, additional processes explaining oncogenic roles of MIRIOOHG have been 
a

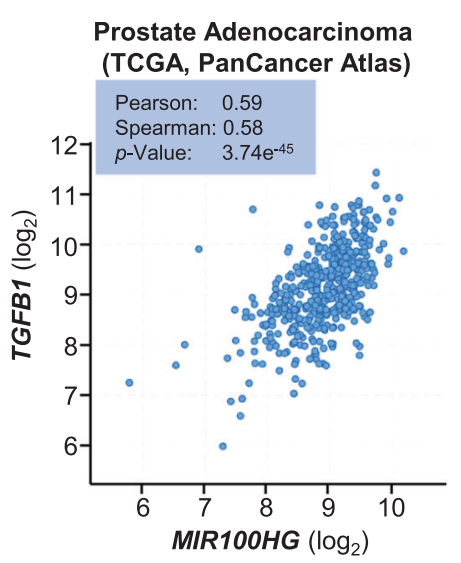

C



\section{e}

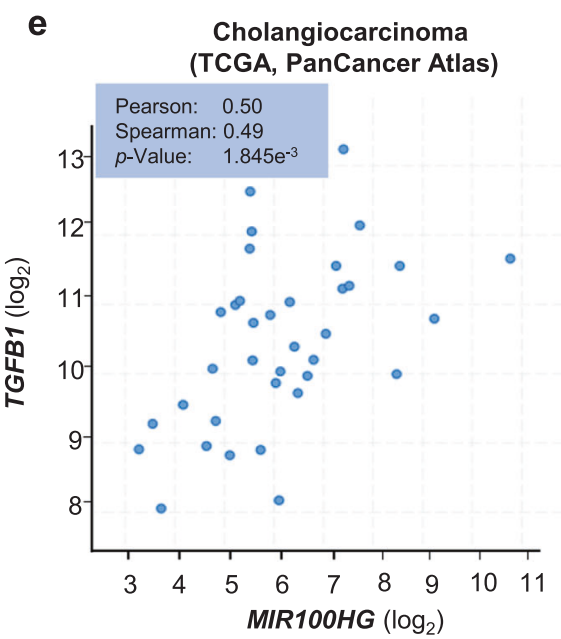

Fig. 8 MIR100HG and TGFBI expression analysis in human cancers. a-e Expression correlation of TGFB1 and MIRIOOHG (a, c, e) or $T G F B I$ and $M I R 100 H G(\mathbf{b}, \mathbf{d})$ in prostate adenocarcinoma (a, b), lung adenocarcinoma (c, d), and cholangiocarcinoma (e) patients enlisted in the PanCancer Atlas. Expression values are reported in a

reported. In acute megakaryoblastic leukemia, silencing of MIR100HG impaired cell proliferation [24]. In osteosarcoma cells, $M I R 100 H G$ expression peaked during early b

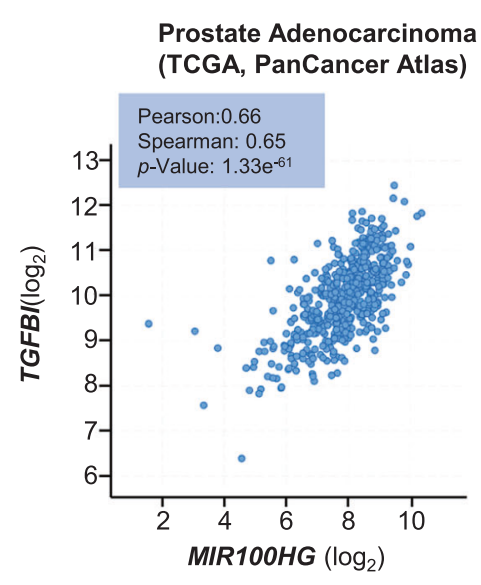

d

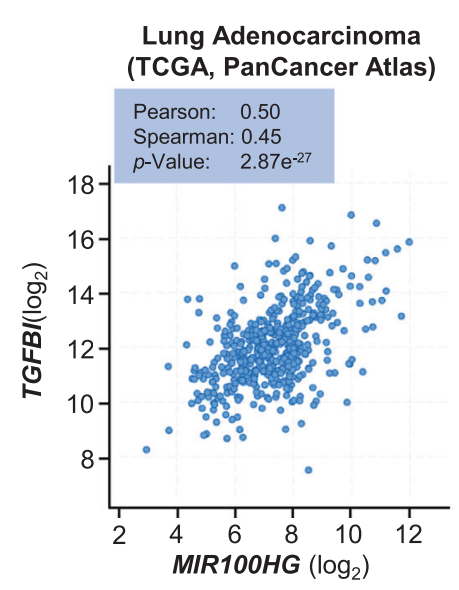

f

Intrahepatic cholangiocarcinoma

(French National Liver Biobank Network)

MIR100HG

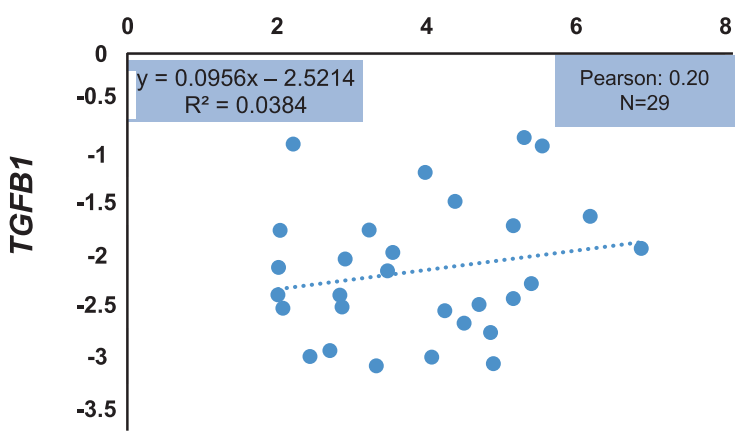

natural logarithmic scale and Pearson and Spearman correlation values are indicated along with the corresponding $P$ values. f Expression correlation of TGFB1 and $M I R 100 H G$ in 29 samples from intrahepatic cholangiocarcinoma patients included in the French national liver biobank network.

G1 cell cycle phase, and $M I R 100 H G$ silencing caused cell cycle arrest [19]. In osteosarcoma cells, MIR100HG promotes proliferation by interacting with the $\mathrm{EZH} 2$ protein of 
the polycomb repressor complex-2, causing repression of LATS1/2, mediators of Hippo signaling [25]. In breast cancer cells, triple-helical formation between MIRIOOHG and $p 27^{\text {Kipl }}$ DNA suppresses $p 27^{\text {Kipl }}$ and promotes proliferation [26].

As TGF $\beta$ signaling instructs the $M I R 100 H G$ promoter, it may also affect the maturation of miRNAs derived from MIR100HG intron-3. TGF $\beta$ regulated expression of some but not all MIR100HG miRNAs with diverse kinetic profiles among different carcinoma cells (Supplementary Figs. S9 and S10). Selective regulation of miRNA maturation could be mediated by the direct association of SMAD3 with specific pre-miRNAs and the enzyme DROSHA [27]. However, MIR100HG-miRNAs were not among those processed via the TGF $\beta / S M A D 3 / D R O S H A-s p e c i f i c$ mechanism [27]. Furthermore, TGF $\beta$ induced expression of $m i R-100$ and $m i R$ $125 \mathrm{~b}$, but not of let-7a-2 in pancreatic adenocarcinoma cells [6]. The distinct MIR100HG-miRNAs induced by TGF $\beta$ in keratinocytes and diverse carcinoma cells (this study) or in pancreatic adenocarcinoma [6] may reflect different physiological outcomes mediated by TGF $\beta$. We suggest that induction of the antitumorigenic let-7a-2-3p by TGF $\beta$ enhances its antiproliferative effect (Supplementary Fig. $\mathrm{S} 14 \mathrm{c})$. On the other hand, in pancreatic adenocarcinoma, TGF $\beta$ frequently loses its antiproliferative power and instead promotes EMT and stemness [28], including enhanced expression of $m i R-100$ and $m i R-125 b$, which promote EMT [6]. Additional studies highlight protumorigenic actions for $m i R-100$ and $m i R-125 b$; chemotherapy-resistant colorectal cancer cells exhibit high MIRI00HG, miR-100, and miR$125 \mathrm{~b}$ expression, the miRNAs enhancing Wnt signaling [29]. In prostate carcinoma PC3U cells, silencing of spliced MIR100HG downregulated expression of mature miR-100 and $m i R-125 b$ (Supplementary Fig. S14c-e). This may explain why silencing MIRIOOHG sensitized the PC3U cells to cytotoxic drugs (Fig. 4d). Furthermore, miR-100 induces mammary EMT by downregulating the chromatin remodeling factor SMARCA5 [30]. We observed that silencing MIRIOOHG reduced TGF $\beta$ responses and overexpression of $M I R 100 H G$, lacking introns and therefore unable to influence intron-3 miRNA biosynthesis (data not shown), resulted in enhanced TGF $\beta$ responses (Figs. 3 and 4). Thus, MIRIOOHG has functions beyond hosting miRNAs in one if its introns.

The link established here between MIR100HG and $T G F B 1$ is supported by analyses of expression in many tumors (Supplementary Fig. S17a). MIR100HG expression data from PanCancer Atlas were reproduced by in situ hybridization in cancer specimen (Supplementary Fig. S17b). Furthermore, clinical data correlated MIR100HG expression with poor survival in certain tumors and with better survival in certain others (Supplementary Fig. S18). Indeed, MIRIO0HG promotes colorectal cancer progression and predicts poor prognosis
[31]. It is possible that the dual action of MIRIOOHG in cancer is linked to the dual action of TGF $\beta$, known to have antitumor properties in certain cancers and protumorigenic actions in others, a matter worth examining in future studies.

\section{Materials and methods}

\section{Reagents}

Recombinant human TGF $\beta 1,1-5 \mathrm{ng} / \mathrm{ml}$ (PeproTech EC Ltd, London, UK) with $4 \mathrm{mM} \mathrm{HCl} / 0.1 \% \mathrm{BSA}$ as a vehicle, GW6604 (5 $\mathrm{MM}$, Ludwig Cancer Research Ltd, New York, USA), LY2157299 (5 $\mu \mathrm{M})$, doxorubicin $(0.7 \mu \mathrm{M})$, taxol $(0.7 \mu \mathrm{M})$, and $5^{\prime}$-fluorouracil $(6.25 \mu \mathrm{M})$ (Sigma-Aldrich, Stockholm, Sweden), with dimethyl sulfoxide as a vehicle, were added to cells.

\section{Plasmids}

Human MIR100HG (NR_024430.2) was amplified using total RNA from MCF10A cells, cDNA synthesis was synthesized via PrimeScript (Takara Bio-Europe, Saint-Germain-en-Laye, France), and PCR-amplified. The cDNA (3129 bp) was cloned in HindIII/EcoRI sites of pcDNA3 (Supplementary Fig. S4a), and sequenced (primers listed in Supplementary Table S1).

\section{Transfections}

SiRNAs/ASOs (20-25 nM; Supplementary Table S2) were transiently transfected once or twice sequentially using siLentFect (Bio-Rad Laboratories, Solna, Sweden) targeting mRNAs or using ASOs; Dharmafect-1 (Dharmacon/VWR, Uppsala, Sweden) targeting lncRNAs; lipofectamine-3000 (ThermoFisherScientific, Stockholm, Sweden) when transfecting plasmids (pcDNA3-MIR100HG, pcDNA3), cotransfecting siRNAs and plasmids or transfecting MirVana miRNA mimics and miRNA inhibitors (Supplementary Table S3; ThermoFisherScientific), each at $10 \mathrm{nM}$. Upon transfection for $24 \mathrm{~h}$, cells switched to starvation medium (0.1-1\% fetal bovine serum (FBS, Biowest, Esbjerg, Denmark) in Dulbecco's modified Eagle's medium (DMEM, Sigma-Aldrich)) and TGF $\beta 1$ stimulation for $24 \mathrm{~h}$ (total period of $48 \mathrm{~h}$ ).

\section{Viability assays}

Cells (3000/well) seeded in 96-well plates, transfected with ASOs or plasmids, were monitored at $48 \mathrm{~h}$ by MTS assay, following the manufacturer's protocol (Promega Biotech AB, Nacka, Sweden). For cytotoxicity analysis, $\mathrm{IC}_{50}$ curves 
for each drug were established in PC3U cells under transfection conditions prior to final experiments. Luminescence units from treated cells were normalized against controls. Graphs show average values of \% viability with standard deviations of at least three biological experiments.

\section{Wound-healing assay}

Cells transfected with ASOs or plasmids for $48 \mathrm{~h}$ were seeded $\left(3 \times 10^{4}\right.$ cells/well $)$ in the complete medium into Culture-Insert-2 (Ibidi GmbH, Gräfelfing, Germany). Confluent cell layers were starved in FBS-free medium for $16 \mathrm{~h}$, the silicone insert was removed, detached cells were removed by washing twice with PBS and vehicle, or $5 \mathrm{ng} / \mathrm{ml}$ TGF $\beta 1$ were added in fresh medium. Wound closure was observed at 0 and $15 \mathrm{~h}$ using a Zeiss Axioplan microscope (objective $\times 10$ ) with MRC digital camera. Wound surface area was quantified by Image $\mathrm{J}-1.47 \mathrm{v}$ as a percentage of open wound per condition.

\section{qPCR, microarray, and database analysis}

RNA extraction, reverse transcription, and $\mathrm{qPCR}$ were performed as described [12] with indicated primers (Supplementary Table S1). Using $\mathrm{RT}^{2}$-Profiler ${ }^{\mathrm{TM}}$ PCR Array (330231/PAHS-035Z, Qiagen, Sollentuna, Sweden), the expression of 84 human TGF $\beta / B M P-S i g n a l i n g-$ Pathway genes was measured. RNA expression was calculated based on the $2^{-\Delta \Delta \mathrm{Ct}}$ method, normalized to reference genes (GAPDH, HPRT1, 18S-rRNA, TBPl) and graphed as averages with standard deviations of at least three biological experiments. Affymetrix transcriptomic data (GSE23952, GSE114761) deposited in the Gene Expression Omnibus (GEO) from NCBI were retrieved and analyzed using the GEO2R web tool. Suitability for direct comparison was assessed by sample value distribution and verification as median-centered. Adjusted $P$ values were calculated via the Benjamini-Hochberg false discovery rate method.

\section{TaqMan assays}

Small ( $<200 \mathrm{nt})$ RNA isolated by the NucleoSpin miRNA kit (Macherey-Nagel, Solna, Sweden) was used for TaqMan advanced miRNA assays (Supplementary Table S4) according to the manufacturer's protocol (ThermoFisherScientific). qPCR was performed on a CFX96 cycler (Bio-Rad Laboratories). miRNA expression was calculated based on the $2^{-\Delta \Delta \mathrm{Ct}}$ method, normalized to reference ( $m i R$ 191-5p, miR-361-5p) miRNAs, and graphed as averages with standard deviations of at least three biological experiments.

\section{In situ hybridization}

Formalin-fixed, paraffin-embedded tissues from melanoma, glioma, and lung adenocarcinoma patients from the Human Protein Atlas project (https://www.proteinatlas.org/) were hybridized in situ for MIR100HG detection using RNAscope Assays (Advanced Cell Diagnostics, Newark, CA, USA) [32].

\section{RNA-binding protein immunoprecipitation}

RIP was performed according to the Magna-RIP ${ }^{\mathrm{TM}}$ RNAbinding protein immunoprecipitation kit (Millipore/Merck, Stockholm, Sweden) as described [12]. Beads loaded with $5 \mu \mathrm{g}$ of anti-HuR antibody (Supplementary Table S5) or normal mouse $\operatorname{IgG}$ (Millipore/Merck) and primers (Supplementary Table S1) were used. Graphs show average values of relative normalized levels (\% input) or enrichment relative to $\operatorname{IgG}$ control with standard deviations of at least three biological experiments.

\section{Luciferase assays}

Luciferase assays in $\mathrm{HaCaT}$ or PC3U cells transiently transfected with the $\mathrm{CAGA}_{12}$-luciferase promoter reporter and siRNA pools, pcDNA3-MIR100HG, or miRNA mimics were performed using the firefly and renilla dualluciferase Assay kit (Biotium, Fremont, CA, USA) as described [12]. Relative normalized luciferase activity is expressed as averages from triplicate determinations, with standard deviations. Each experiment was repeated at least twice.

\section{ChIP}

ChIP experiments were performed as described [12], with $3 \mu \mathrm{g}$ of anti-Smad2/3 antibody (BD Biosciences-Europe, Stockholm, Sweden), normal mouse IgG (Millipore/Merck), and primers for qPCR of precipitated MIRIOOHG and SERPINE1 DNAs (Supplementary Table S1).

\section{Immunoblotting}

Protein extraction, nucleocytoplasmic fractionation followed by RNA extraction, protein quantification, and immunoblotting was performed as described [12], with primary antibodies (Supplementary Table S5) and densitometric quantification performed using ImageJ-1.47 (National Institutes of Health, MD, USA). Protein-band density normalized against the corresponding loading control ( $\alpha$-TUBULIN, $\beta$-ACTIN, or GAPDH) is expressed as 1 under basal or control conditions. Phosphoprotein and 
cleaved-protein density was normalized to the corresponding total protein.

\section{Thymidine-incorporation assay}

HaCaT cells transiently transfected with siRNAs or miRNA mimics were seeded in $1 \%$ FBS/DMEM and treated with TGF $\beta 1$ for $24 \mathrm{~h}$. Thymidine-incorporation assays were performed as described [12]. Average values with standard deviation of triplicate repeats for each condition are plotted from experiments repeated twice.

\section{ELISA}

PC3U or A549-conditioned media were concentrated 50x through Amicon Ultra-15 centrifugal filters (Merck/Millipore) at $3000 \times g$ for $15 \mathrm{~min}$ at $4{ }^{\circ} \mathrm{C}$ or used without concentration (for certain PC3U experiments). Secreted mature TGF $\beta 1$ was measured using the human TGF $\beta 1$-Duoset ELISA kit according to the manufacturer's instructions (R\&D Systems, Oxon, UK).

\section{PanCancer Atlas and cholangiocarcinoma cohort analysis}

The cBioPortal for Cancer Genomics [33, 34] was used to retrieve RNA-seq data from cancer patients and gene coexpression analyses of MIR $100 H G$ in carcinomas. All gene expressions were equally weighted. For MIRIOOHG$T G F B 1$ coexpression analysis in the intrahepatic cholangiocarcinoma cohort from the French national liver biobank network, tissues were acquired as described [35], after written informed consent from all patients and study protocol approval by the local ethics committee and institutional review board of INSERM (IRB00003888). Pearson correlation value was calculated as described [35]. Survival Kaplan-Meier plots were generated using the OncoLnc platform [22].

\section{miRNA target analysis}

Targets of let-7a-5p and let-7a-2-3p miRNAs were predicted using the DIANA microT-CDS algorithm [20]. For pathway prediction among let-7a-2-3p targets, DIANA mirPathv. 3 was used ( $p$-value threshold 0.001 , microT threshold 0.8). For KEGG pathway prediction of common and unique targets of let-7a-5p and let-7a-2-3p, Enrichr (https://amp.pharm.mssm.edu/Enrichr/\#) was used.

\section{Cell culture}

Cell and media information is listed in Supplementary Table S6. Cells were free of mycoplasma (tested every 2 months) and authenticated using PCR single-locus technology (Eurofins, Uppsala, Sweden).

\section{Statistics}

The results are shown as mean values from $n=3$ or $n=2$ independent biological experiments. Error bars represent standard deviations. Each biological experiment included triplicate or quintuplicate technical repeats. Comparisons were performed using a two-tailed paired Student's $t$ test and statistical significance is represented by stars $(* P<0.05$, $* * P<0.01, * * * P<0.001)$.

Acknowledgements We thank members of our laboratory for discussions, Caroline Gélabert and Eric Ahlström for technical assistance, and Anders Sundqvist for advice and reagents.

Author contributions PP and ArM conceived the project; PP and DMRJr designed experiments; PP, DMRJr, AnM, AB, and LC acquired the data; PP, DMRJr, LC, and ArM analyzed the data; PP, DMRJr, AnM, LC, CC, FP, CHH, and ArM interpreted the data; PP drafted and ArM finalized the paper. All authors critically revised the paper and approved submission for publication.

Funding This work was supported by Ludwig Cancer Research, Cancerfonden [CAN2012/438, CAN2015/438, CAN2018/469] to ArM, Vetenskapsrådet [K2013-66X-14936-10-5, 2017-01588-3, 2018-02757-3] to ArM, [2015-02757, 2020-01291] to CHH, Barncancerfonden [PR2018-0091] to ArM, European Research Council [787472] to $\mathrm{CHH}$, Bodossaki Foundation and Alexander Onassis Foundation, Greece to PP, Inserm, Université de Rennes-1, and ITMO Cancer AVIESAN Plan Cancer [C18007NS] to CC.

\section{Compliance with ethical standards}

Compliance with ethical standards The intrahepatic cholangiocarcinoma cohort from the French national liver biobank network has been maintained after written informed consent from all patients and study protocol approval by the local ethics committee and institutional review board of INSERM (IRB00003888).

Conflict of interest The authors declare no competing interests.

Publisher's note Springer Nature remains neutral with regard to jurisdictional claims in published maps and institutional affiliations.

Open Access This article is licensed under a Creative Commons Attribution 4.0 International License, which permits use, sharing, adaptation, distribution and reproduction in any medium or format, as long as you give appropriate credit to the original author(s) and the source, provide a link to the Creative Commons license, and indicate if changes were made. The images or other third party material in this article are included in the article's Creative Commons license, unless indicated otherwise in a credit line to the material. If material is not included in the article's Creative Commons license and your intended use is not permitted by statutory regulation or exceeds the permitted use, you will need to obtain permission directly from the copyright holder. To view a copy of this license, visit http://creativecommons. org/licenses/by/4.0/. 


\section{References}

1. Esteller M. Non-coding RNAs in human disease. Nat Rev Genet. 2011;12:861-74.

2. Pelechano V, Steinmetz LM. Gene regulation by antisense transcription. Nat Rev Genet. 2013;14:880-93.

3. Tordonato C, Di Fiore PP, Nicassio F. The role of non-coding RNAs in the regulation of stem cells and progenitors in the normal mammary gland and in breast tumors. Front Genet. 2015;6:72.

4. Dews M, Fox JL, Hultine S, Sundaram P, Wang W, Liu YY, et al. The myc-miR-17 92 axis blunts TGF $\beta$ signaling and production of multiple TGF $\beta$-dependent antiangiogenic factors. Cancer Res. 2010;70:8233-46.

5. Montes M, Nielsen MM, Maglieri G, Jacobsen A, Hojfeldt J, Agrawal-Singh $\mathrm{S}$, et al. The lncRNA MIR31HG regulates p16INK4A expression to modulate senescence. Nat Commun. 2015;6:6967.

6. Ottaviani S, Stebbing J, Frampton AE, Zagorac S, Krell J, de Giorgio A, et al. TGF- $\beta$ induces miR-100 and miR-125b but blocks let-7a through LIN28B controlling PDAC progression. Nat Commun. 2018;9:1845.

7. Ha M, Kim VN. Regulation of microRNA biogenesis. Nat Rev Mol cell Biol. 2014;15:509-24.

8. Bueno MJ, Malumbres M. MicroRNAs and the cell cycle. Biochim Biophys Acta. 2011;1812:592-601.

9. David CJ, Massagué J. Contextual determinants of TGF $\beta$ action in development, immunity and cancer. Nat Rev Mol Cell Biol. 2018;19:419-35.

10. Papoutsoglou P, Moustakas A. Long non-coding RNAs and TGF$\beta$ signaling in cancer. Cancer Sci. 2020;111:2672-81.

11. Heldin C-H, Landström M, Moustakas A. Mechanism of TGF- $\beta$ signaling to growth arrest, apoptosis, and epithelial-mesenchymal transition. Curr Opin Cell Biol. 2009;21:166-76.

12. Papoutsoglou P, Tsubakihara Y, Caja L, Morén A, Pallis P, Ameur A, et al. The TGFB2-AS1 lncRNA regulates TGF- $\beta$ signaling by modulating corepressor activity. Cell Rep. 2019;28:3182-98. e3111

13. Maupin KA, Sinha A, Eugster E, Miller J, Ross J, Paulino V, et al. Glycogene expression alterations associated with pancreatic cancer epithelial-mesenchymal transition in complementary model systems. PLoS ONE. 2010;5:e13002.

14. Gordian E, Welsh EA, Gimbrone N, Siegel EM, Shibata D, Creelan BC, et al. Transforming growth factor $\beta$-induced epithelial-to-mesenchymal signature predicts metastasis-free survival in non-small cell lung cancer. Oncotarget. 2019;10:810-24.

15. Ma WJ, Cheng S, Campbell C, Wright A, Furneaux H. Cloning and characterization of $\mathrm{HuR}$, a ubiquitously expressed Elav-like protein. J Biol Chem. 1996;271:8144-51.

16. Spångberg K, Wiklund L, Schwartz S. HuR, a protein implicated in oncogene and growth factor mRNA decay, binds to the $3^{\prime}$ ends of hepatitis $\mathrm{C}$ virus RNA of both polarities. Virology. 2000;274:378-90.

17. Bai D, Gao Q, Li C, Ge L, Gao Y, Wang H. A conserved TGF $\beta 1 /$ HuR feedback circuit regulates the fibrogenic response in fibroblasts. Cell Signal. 2012;24:1426-32.

18. Nabors LB, Gillespie GY, Harkins L, King PH. HuR, a RNA stability factor, is expressed in malignant brain tumors and binds to adenine- and uridine-rich elements within the $3^{\prime}$ untranslated regions of cytokine and angiogenic factor mRNAs. Cancer Res. 2001;61:2154-61.
19. Sun Q, Tripathi V, Yoon JH, Singh DK, Hao Q, Min KW, et al. MIR100 host gene-encoded lncRNAs regulate cell cycle by modulating the interaction between HuR and its target mRNAs. Nucleic Acids Res. 2018;46:10405-16.

20. Vlachos IS, Hatzigeorgiou AG. Functional analysis of miRNAs using the DIANA tools online suite. Methods Mol Biol. 2017;1517:25-50.

21. Hoadley KA, Yau C, Hinoue T, Wolf DM, Lazar AJ, Drill E, et al. Cell-of-origin patterns dominate the molecular classification of 10,000 tumors from 33 types of cancer. Cell. 2018;173:291-304. e296

22. Anaya J. OncoLnc: linking TCGA survival data to mRNAs, miRNAs, and lncRNAs. Peerj Comput Sci. 2016;2:e67.

23. Pabis M, Popowicz GM, Stehle R, Fernandez-Ramos D, Asami S, Warner L, et al. HuR biological function involves RRM3mediated dimerization and RNA binding by all three RRMs. Nucleic Acids Res. 2019;47:1011-29.

24. Emmrich S, Streltsov A, Schmidt F, Thangapandi VR, Reinhardt D, Klusmann JH. LincRNAs MONC and MIR100HG act as oncogenes in acute megakaryoblastic leukemia. Mol Cancer. 2014;13:171.

25. Su X, Teng J, Jin G, Li J, Zhao Z, Cao X, et al. ELK1-induced upregulation of long non-coding RNA MIR100HG predicts poor prognosis and promotes the progression of osteosarcoma by epigenetically silencing LATS1 and LATS2. Biomed Pharmacother. 2019;109:788-97.

26. Wang $\mathrm{S}, \mathrm{Ke} \mathrm{H}$, Zhang $\mathrm{H}$, Ma Y, Ao L, Zou L, et al. LncRNA MIR100HG promotes cell proliferation in triple-negative breast cancer through triplex formation with p27 loci. Cell Death Dis. 2018;9:805.

27. Davis BN, Hilyard AC, Nguyen PH, Lagna G, Hata A. Smad proteins bind a conserved RNA sequence to promote microRNA maturation by Drosha. Mol Cell. 2010;39:373-84.

28. David CJ, Huang YH, Chen M, Su J, Zou Y, Bardeesy N, et al. TGF- $\beta$ tumor suppression through a lethal EMT. Cell. 2016;164:1015-30.

29. Lu Y, Zhao X, Liu Q, Li C, Graves-Deal R, Cao Z, et al. IncRNA MIR100HG-derived miR-100 and miR-125b mediate cetuximab resistance via Wnt/ $\beta$-catenin signaling. Nat Med. 2017;23:1331-41.

30. Chen D, Sun Y, Yuan Y, Han Z, Zhang P, Zhang J, et al. miR-100 induces epithelial-mesenchymal transition but suppresses tumorigenesis, migration and invasion. PLoS Genet. 2014;10:e1004177.

31. Li W, Yuan F, Zhang X, Chen W, Tang X, Lu L. Elevated MIR100HG promotes colorectal cancer metastasis and is associated with poor prognosis. Oncol Lett. 2019;18:6483-90.

32. Wang F, Flanagan J, Su N, Wang LC, Bui S, Nielson A, et al. RNAscope: a novel in situ RNA analysis platform for formalin-fixed, paraffin-embedded tissues. J Mol Diagnostics. 2012;14:22-29.

33. Cerami E, Gao J, Dogrusoz U, Gross BE, Sumer SO, Aksoy BA, et al. The cBio cancer genomics portal: an open platform for exploring multidimensional cancer genomics data. Cancer Discov. 2012;2:401-4.

34. Gao J, Aksoy BA, Dogrusoz U, Dresdner G, Gross B, Sumer SO, et al. Integrative analysis of complex cancer genomics and clinical profiles using the cBioPortal. Sci Signal. 2013;6:pl1.

35. Merdrignac A, Angenard G, Allain C, Petitjean K, Bergeat D, Bellaud $\mathrm{P}$, et al. A novel transforming growth factor $\beta$-induced long noncoding RNA promotes an inflammatory microenvironment in human intrahepatic cholangiocarcinoma. Hepatol Commun. 2018;2:254-69. 